\title{
INFRA-RED SPECTRA OF NEON, ARGON, AND KRYPTON
}

\author{
By William F. Meggers and C. J. Humphreys
}

ABSTRACT

The first spectra of neon, argon, and krypton have been photographed in the infra-red (7,600 to 12,200 A) with two new Eastman emulsions having $\mathrm{P}$ and $\mathrm{Q}$ types of sensitization with sensitivity maxima at 8,600 and 9,700 A, respectively. The sources were Geissler tubes operated with uncondensed high-voltage a. c. discharges, and the spectrographs were concave gratings of 21 feet radius. About 200 infra-red lines have been photographed in the spectrum of each of the noble gases and analysis has resulted in the confirmation and extension of the tables of spectral terms. Several new hydrogen-like terms from $f$-type electrons have been found in each spectrum, and the $3 d$ terms lacking in the A I and Kr I spectra have all been identified. Many of the new lines can be accurately computed from relative terms and such calculated values may serve as preliminary standards of wave lengths in the infra-red.

CONTENTS

I. Introduction

II. Wave-length measurements III. Results

1. Neon.

2. Argon

3. Krypton

\section{INTRODUCTION}

Neutral atoms of the noble gases are characterized by spectra of exceptional utility and rare beauty. Geissler tubes containing these gases at low pressure when excited by moderate electrical discharges, emit spectral lines of high homogeneity which have been demonstrated to be reproducible within 1 part in 50,000,000. These lines are favorably regarded as standards of wave length, and are extensively used in spectroscopy and metrology. It is not unlikely that one of these lines may ultimately be chosen as the most practical and permanent primary standard of length, especially if an even-numbered isotope can be segregated so that the strongest lines will be free from hyperfine structure.

From a theoretical viewpoint the first. spectra of the noble gases are of special interest because they arise in each case from a "closed shell" configuration of electrons, and unlike other complex spectra they are found to consist of extended series of relatively simple types (S,P,D,F terms) and low multiplicities (singlets and triplets). Evidence of a gradual transition from $s l$ to $j j$ electron coupling as the atomic complexity increases adds interest to a comparison of the spectral structures of all the noble gases.

Both practical and theoretical considerations have been responsible for intensive investigations of the noble gas spectra, and especially for efforts to extend observations to the region of greater wave lenghts. Except for a relatively small number of lines in the extreme ultraviolet, each of these spectra exhibits several group series beginning in the red or infra-red and converging to limits in the near ultra-violet or in the visible region. The fact that many of the intrinsically strongest lines of these spectra occur in the infra-red accounts for the 
successful photography of a considerable proportion of them even with relatively insensitive materials. Thus the most energetic infra-red lines of Ne, A, Kr, and Xe had already been detected photographically to about $1 \mu$ with dicyanine or neocyanine sensitized plates when about a year ago the vastly superior infra-red sensitizers, mesocyanine and xenocyanine, were discovered. As soon as the new photographic plates became available for spectrography, the spectroscopy section of this bureau applied them to explorations of infra-red emission spectra, including those characteristic of the noble gases. Preliminary results on this group were reported ${ }^{1}$ at a meeting of the American Physical Society last April. In the meantime the observations have been repeated and extended, and details have been published for two of these elements, viz, $\mathrm{He}^{2}$ and $\mathrm{Xe}^{3}$ Data for the intermediate gases, $\mathrm{Ne}, \mathrm{A}$, and $\mathrm{Kr}$, are now presented, they consist of wave-length measurements, estimated intensities and series classifications of infra-red lines in the wave length interval 7,600 to $12,200 \mathrm{~A}$. These results are to be regarded as preliminary or exploratory; they were obtained with spectrographs of moderate dispersion, but the accuracy of measurement is sufficient for purposes of spectral structure analysis, and for the determination of retardations in future interferometric comparisons. Wave lengths corresponding to the stronger lines are being measured with increased precision by the Fabry-Perot interferometer method and will be presented in a subsequent paper on standard wave lengths in the infra-red. In the meantime values computed from accurately fixed relative terms may serve as proper standards in this range, since the accuracy of such calculated values may be expected to exceed that of the preliminary observations.

The spark spectra of the heavier noble gases are also rich in infrared lines, but their description is reserved for later publication.

\section{WAVE-LENGTH MEASUREMENTS}

Geissler tubes of glass purchased from Robert Goetze in Leipzig served as sources of radiation. Each tube has two cylindrical electrode bulbs connected by a capillary of $11 \mathrm{~cm}$ length and about 1 $\mathrm{mm}$ bore, the viewing end of which is inclosed in a thin-walled glass bulb of $2.5 \mathrm{~cm}$ diameter. Such tubes can be viewed either side on or end on, the latter being preferred on account of increased intensity. In the experiments here reported the tube was always mounted with its capillary on the axis of the spectrograph with the viewing end directed toward the slit, the capillary bore being imaged on the slit with a condensing lens.

The tubes were filled with exceptionally pure gases at sufficient pressure to insure relatively long life in spite of gradual gas disappearance accompanying electrode sputtering. To produce the first spectrum the tube was operated with uncondensed electrical discharges from a. c. transformers, the current through the tube being about 20 ma. Under these conditions no spark lines appeared in the spectral interval under investigation. Spectrochemical analysis revealed a trace of $\mathrm{O}$ in the $\mathrm{Ne}$, no impurities in the $\mathrm{A}$, a small amount of $\mathrm{A}$ and $\mathrm{Xe}$ in the $\mathrm{Kr}$ and a trace of $\mathrm{Kr}$ in the $\mathrm{Xe}$.

\footnotetext{
1 W. F. Meggers and C. J. Humphreys, Phys. Rev., vol. 40, p. 1040, 1932.

2 W. F. Meggers and G. H. Dieke, B. S. Jour. Research, vol. 9 (RP462), p. 121, 1932.

C. J. Humphreys and W. F. Meggers, B. S. Jour. Research, vol. 10, (R P521) p. 139, 1933.

- End-on use of these sources is objectionable in interferometry on account of self-reversal phenomena then appearing on lines involving normal or metastable states.
} 
The spectrograms were made with two concave diffraction gratings of 21 feet radius of curvature, each mounted in parallel light from a mirror of similar radius. ${ }^{5}$ One grating has 20,000 lines per inch and the other 7,500 lines per inch, their scales in the first-order red spectrum are 3.7 and $10.2 \mathrm{~A} / \mathrm{mm}$, respectively. The larger dispersion was applied to the interval 7,600 to $9,200 \mathrm{~A}$, while the smaller scale was used over the entire range 7,600 to $12,200 \mathrm{~A}$. On each spectrogram the spectrum of the iron arc was recorded in the second and third orders of the grating alongside the spectrum of the noble gas which was photographed in the first order with a red glass filter in front of the slit to remove overlapping higher orders. Wave-length measurements were then made relative to international standards in the iron spectrum, doubling or trebling their values to obtain effective standards in the infra-red range. The final scale of these measurements was adjusted slightly to bring it into accord with preliminary values derived from interferometer comparisons of the stronger lines. Wave-length measurements from the larger scale spectrograms should not contain errors exceeding one or two hundredths of an angstrom unit, but the remainer may have an average error two or three times as large.

Exposure times were usually 16 to 24 hours, but one 48 -hour exposure was made for each gas in order to record as many lines as possible beyond $11,000 \mathrm{~A}$ where the photographic sensitivity is very low. Under these conditions the stronger lines were tremendously overexposed, and were accompanied by numerous Rowland ghosts from which the wave length of the parent line could be derived with higher accuracy than from measurements of the greatly widened primary image. All such ghosts were removed from the final lists. Lyman ghosts ${ }^{6}$ were sought but only one was found, a line observed for $\mathrm{Kr}$ at $12,282 \mathrm{~A}$ being interpreted as $(7 / 5-1 / 1,500) 8,776.75 \mathrm{~A}$.

Photographic plates with $\mathrm{P}$ and $\mathrm{Q}$ types of sensitizing as prepared by the research laboratory of the Eastman Kodak Co. ${ }^{7}$ were employed. These plates are sensitized to infra-red radiation with new dyes which have been named mesocyanine and xenocyanine. The former has a relatively narrow sensitizing band with a strong maximum at 8,600 $A$, while the latter has a much broader band with a maximum action at wave length 9,700 A. The P-type plates were used in the interval 7,600 to $9,200 \mathrm{~A}$, and the Q-type in the range 8,500 to $12,200 \mathrm{~A}$. Both types of plates were hypersensitized by bathing from 1 to 5 minutes in a dilute ammonia solution, rinsing in alcohol, and drying in a current of air. After exposure they were developed for 5 minutes in Eastman's X-ray developer at a temperature of about $18^{\circ} \mathrm{C}$.

\section{RESULTS}

\section{NEON}

Paschen's ${ }^{8}$ description and analysis of the spectrum of neon has served as a model for the remaining noble gases. A special notation which was proposed by Paschen to represent the spectral terms of neon has been retained in describing the first spectrum of the remaining gases of this group. A translation into the notation now in general use, while highly desirable, is not feasible at present, because of the

W. F. Meggers and Keivin Burns, B. S. Sci. Papers, vol. 18 (S441), p. 191, 1922.

W. F. Meggers and C. C. Kiess, J. Opt. Soc. Am. and Rev. Sci. Inst., vol. 6, p. 417, 1922.

7 C. E. K. Mees, J. Opt. Soc. Am. vol. 22, p. 204, 1932. Addendum, April, 1932.

8 F. Paschen, Ann. der Physik, vol, 60, p. 405, 1919. 
overlapping of the multiplets, the fact that the Landé interval rule and theoretical Zeeman effects are usually violated in these spectra, and on account of ambiguities in the correlation of levels with their series limits. Nothing was added to the results for neon until Gremmer ${ }^{9}$ found some new lines by exposing neocyanine plates 36 to 60 hours. These additional lines fixed, by means of new combinations, four $2 \mathrm{~s}$ terms for which only series interpolated values could be given by Paschen. Altogether Gremmer added 27 classified lines to the infrared between 7,944.22 and 9,665.40 A. By employing an efficient spectograph, an intense source, and very long exposures, Rasmussen ${ }^{10}$ recorded on neocyanine plates 7 new lines in the neon spectrum $(10,296$ to $11,178 \mathrm{~A})$, which were classified as $2 \mathrm{p}-2 \mathrm{~s}$ combinations.

The infra-red spectrum of neon was investigated with a spectrometer, thermopile, and galvanometer by Hardy ${ }^{11}$, who observed 30 lines ranging in wave length from $9,667.6$ to $18,552 \mathrm{~A}$. We have recorded 16 of these lines photographically, the last one being 12,066.4 $A$, and have adopted Hardy's values for the remainder, with a correction of $-2.6 \mathrm{~A}$ applied to them, since the lines observed by both methods differ by this amount on the average. Comparison of galvanometer deflections with estimated photographic intensities gives a rough calibration of the spectral sensitivity of a xenocyanine plate, at $11,000 \mathrm{~A}$ the sensitivity is less than one-tenth that of the maximum near 9,700 A and at 12,000 A it has declined to less than one one-thousandth.

Complete results now available for the infra-red spectrum of neon are presented in Table 1, where the intensity, observed wave length, vacuum wave number, symbolic-term difference, and numerical-term difference appear in successive columns. Wave lengths given to 3 decimals are quoted from Paschen's publication; they represent interferometer measurements of high precision. The vacuum wave numbers were taken from Kayser's ${ }^{12}$ table for wave lengths up to $10,000 \mathrm{~A}$, beyond which they were obtained by computing the reciprocal of the wave lengths corrected to vacuum values with the aid of the atmospheric dispersion formula of Meggers and Peters. ${ }^{13}$

TABLE 1.-Infra-red spectrum of neon

\begin{tabular}{|c|c|c|c|c|}
\hline Intensity & $\lambda_{\mathrm{air}}$ I. A. & $\begin{array}{l}\nu_{\mathrm{vac}} \mathrm{cm}^{-1} \\
\text { observed }\end{array}$ & $\begin{array}{l}\text { Term combi- } \\
\text { nation }\end{array}$ & $\begin{array}{l}\nu_{\mathrm{vac}} \mathrm{cm}^{-1} \\
\text { calculated }\end{array}$ \\
\hline Hardy & Hardy & & \multirow{4}{*}{$\begin{array}{c}3 d_{1}^{\prime}-4 \mathrm{~W} \\
3 s_{1}^{\prime \prime \prime}-4 \mathrm{~V} \\
\left\{3 s_{1}^{\prime \prime \prime}-4 \mathrm{~T}\right. \\
3 s_{1}^{\prime \prime \prime \prime}-4 U \\
3 d_{3}-4 \mathrm{Y}\end{array}$} & \multirow{4}{*}{$\begin{array}{l}5,390 \\
5,425.2 \\
5,437.2 \\
5,438.1 \\
5,462.02\end{array}$} \\
\hline $\begin{array}{l}1 \\
1\end{array}$ & $\begin{array}{l}18,549 \\
18,430\end{array}$ & $\begin{array}{l}5,389.7 \\
5,424.5\end{array}$ & & \\
\hline 2 & $18,386.4$ & $5,437.32$ & & \\
\hline 1 & $18,306.4$ & $5,461.08$ & & \\
\hline 3 & $18,271.4$ & $5,471.54$ & $3 d_{3}-4 \mathrm{Z}$ & 5,471 \\
\hline 1 & $18,078.4$ & $5,529.96$ & $3 d_{5}-4 X$ & $5,528.50$ \\
\hline 1 & $17,165.4$ & $5,824.08$ & $2 p_{1}-2 s_{4}$ & $5,825.25$ \\
\hline 2 & $15,234.4$ & $6,562.28$ & $2 p_{1}-2 s_{2}$ & $6,563.85$ \\
\hline 1 & $13,219.4$ & $7,562.58$ & $2 p_{2}-2 s_{5}$ & $7,562.69$ \\
\hline
\end{tabular}

W. Gremmer, Zeitschr. f. Phys., vol. 50, p. 716, 1928.

10 F. Rasmussen, Dissertation, Çopenhagen, 1932.

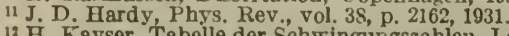

12 H. Kayser, Tabelle der Schwingungszahlen, Leipzig, 1925.

13 W. F. Meggers and C. G. Peters, B. S. Sci. Papers, vol. 14 (S327), p. 722, 1918. 
TABLE 1.-Ir.fra-red spectrum of neon-Continued

\begin{tabular}{|c|c|c|c|c|}
\hline Intensity & $\lambda_{\mathrm{ai}, \mathrm{I}}$ I. A. & $\begin{array}{l}\nu_{\text {vac }} \mathrm{cm}^{-1} \\
\text { observed }\end{array}$ & $\begin{array}{l}\text { Term combi- } \\
\text { nation }\end{array}$ & $\begin{array}{l}\nu_{\text {vac }} \mathrm{cm}^{-1} \\
\text { calculated }\end{array}$ \\
\hline $\begin{array}{l}\text { Hardy } \\
2 \\
0.3 \\
2 \\
1 \\
1\end{array}$ & $\begin{array}{c}\text { Hardy } \\
12,912.4 \\
12,767.4 \\
12,690.1 \\
12,594.8 \\
12,458.4\end{array}$ & $\begin{array}{l}7,742.40 \\
7,830.30 \\
7,877.99 \\
7,937.64 \\
8,024.52\end{array}$ & $\begin{array}{l}2 p_{4}-2 s_{5} \\
2 p_{5}-2 s_{5} \\
2 p_{3}-2 s_{4} \\
2 p_{4}-2 s_{4} \\
2 p_{5}-2 s_{4}\end{array}$ & $\begin{array}{l}7,742.63 \\
7,829.03 \\
7,878.54 \\
7,937.47 \\
8,023.87\end{array}$ \\
\hline $\begin{array}{c}\text { B. S. } \\
1 \\
1 \\
3 \\
4 \\
1\end{array}$ & $\begin{array}{c}\text { B. S. } \\
12,066.4 \\
11,985.1 \\
11,789.0 \\
11,766.9 \\
11,688.1\end{array}$ & $\begin{array}{l}8,285.21 \\
8,341.40 \\
8,480.17 \\
8,496.10 \\
8,553.37\end{array}$ & $\begin{array}{l}2 p_{6}-2 s_{5} \\
2 p_{2}-2 s_{3} \\
2 p_{6}-2 s_{4} \\
2 p_{2}-2 s_{2} \\
2 p_{1}-3 d_{5}\end{array}$ & $\begin{array}{l}8,285.28 \\
8,341.53 \\
8,480.12 \\
8,496.13 \\
8,553.485\end{array}$ \\
\hline $\begin{array}{l}3 \\
2 \\
4 \\
8 \\
4\end{array}$ & $\begin{array}{l}11,614.20 \\
11,536.35 \\
11,525.10 \\
11,522.83 \\
11,409.27\end{array}$ & $\begin{array}{l}8,607.80 \\
8,665.89 \\
8,674.34 \\
8,676.05 \\
8,762.41\end{array}$ & $\begin{array}{l}2 p_{5}-2 s_{3} \\
2 p_{1}-3 d_{2} \\
2 p_{7}-2 s_{4} \\
2 p_{1}-2 s_{2} \\
2 p_{5}-2 s_{2}\end{array}$ & $\begin{array}{l}8,607.87 \\
8,665.865 \\
8,674.38 \\
8,676.07 \\
8,762.47\end{array}$ \\
\hline $\begin{array}{r}5 \\
15 \\
30 \\
1 \\
3\end{array}$ & $\begin{array}{l}11,390.50 \\
11,177.51 \\
11,142.04 \\
11,120.3 \\
11,049.6\end{array}$ & $\begin{array}{l}8,776.85 \\
8,944.09 \\
8,971.76 \\
8,990.11 \\
9,047.63\end{array}$ & $\begin{array}{l}2 p_{8}-2 s_{5} \\
2 p_{9}-2 s_{5} \\
2 p_{8}-2 s_{4} \\
2 s_{2}-4 p_{8} \\
2 s_{5}-4 p_{8}\end{array}$ & $\begin{array}{l}8,776.92 \\
8,944.10 \\
8,971.76 \\
8,990.16 \\
9,047.60\end{array}$ \\
\hline $\begin{array}{r}2 \\
2 \\
3 \\
2 \\
200\end{array}$ & $\begin{array}{l}11,044.1 \\
11,020.8 \\
10,888.4 \\
10,886.1 \\
10,844.47\end{array}$ & $\begin{array}{l}9,052.13 \\
9,071.27 \\
9,181.57 \\
9,183.51 \\
9,218.77\end{array}$ & $\begin{array}{l}2 s_{2}-4 p_{1} \\
2 s_{4}-4 p_{3} \\
3 d_{1}^{\prime}-6 Z \\
3 d_{1}^{\prime \prime}-6 Z \\
2 p_{6}-2 s_{2}\end{array}$ & $\begin{array}{l}9,052.43 \\
9,071.32 \\
9,181.6 \\
9,183.4 \\
9,218.72\end{array}$ \\
\hline $\begin{array}{l}4 \\
2 \\
1 \\
2 \\
2\end{array}$ & $\begin{array}{l}10,830.33 \\
10,819.9 \\
10,814.7 \\
10,808.1 \\
10,806.3\end{array}$ & $\begin{array}{l}9,230.80 \\
9,239.70 \\
9,244.1 \\
9,249.8 \\
9,251.3\end{array}$ & $\begin{array}{l}3 s_{1}{ }^{\prime \prime}-6 \mathrm{U} \\
3 d_{2}-6 \mathrm{Y} \\
3 s_{1}^{\prime \prime \prime}-6 \mathrm{U} \\
3 s_{1}^{\prime \prime \prime \prime}-6 \mathrm{U}\end{array}$ & $\begin{array}{l}9,239.92 \\
9,244.2 \\
9,249.68 \\
9,251.24\end{array}$ \\
\hline $\begin{array}{c}100 \\
2 \\
3 \\
4 \\
1 ?\end{array}$ & $\begin{array}{l}10,798.02 \\
10,780.40 \\
10,766.03 \\
10,763.98 \\
10,760.4\end{array}$ & $\begin{array}{l}9,258.42 \\
9,273.56 \\
9,285.93 \\
9,287.70 \\
9,290.8\end{array}$ & $\begin{array}{l}2 p_{T}-2 s_{3} \\
3 d_{3}-7 Y \\
3 d_{1}^{\prime}-6 W \\
3 d_{1}^{\prime \prime}-6 W \\
3 d_{4}-6 Z\end{array}$ & $\begin{array}{l}9,258.38 \\
9,273.6 \\
9,285.93 \\
9,287.70 \\
9,290.9\end{array}$ \\
\hline $\begin{array}{c}1 ? \\
3 \\
1 \\
30 \\
300\end{array}$ & $\begin{array}{l}10,758.2 \\
10,690.35 \\
10,673.7 \\
10,620.63 \\
10,562 \cdot 38\end{array}$ & $\begin{array}{l}9,292.7 \\
9,351.67 \\
9,366.3 \\
9,413.01 \\
9,564.97\end{array}$ & $\begin{array}{l}3 d_{4}{ }^{\prime}-6 \mathrm{Z} \\
3 d_{5}^{\prime}-6 \mathrm{X} \\
3 d_{6}-6 \mathrm{X} \\
2 p_{T}-2 s_{2} \\
2 p_{1}-3 s_{1}{ }^{\prime}\end{array}$ & $\begin{array}{l}9,292.7 \\
9,351.64 \\
9,366.28 \\
9,412.98 \\
9,464.941\end{array}$ \\
\hline $\begin{array}{r}3 \\
80 \\
1 \\
7 \\
2\end{array}$ & $\begin{array}{l}10,432.53 \\
10,295.40 \\
10,267.0 \\
10,245.70 \\
10,224.6\end{array}$ & $\begin{array}{l}9,582.77 \\
9,710.41 \\
9,737.28 \\
9,757.51 \\
9,777.65\end{array}$ & $\begin{array}{l}2 s_{4}-4 p_{4} \\
2 p-2 s_{2} \\
2 s_{5}-4 p_{2} \\
2 s_{5}-4 p_{4}\end{array}$ & $\begin{array}{l}9,582.82 \\
9,710.36 \\
9,757.56 \\
9,777.66\end{array}$ \\
\hline $\begin{array}{l}2 \\
3 \\
1 \\
2 \\
2\end{array}$ & $\begin{array}{l}10,210.73 \\
10,091.53 \\
10,057.6 \\
10,038.9 \\
10,037.1\end{array}$ & $\begin{array}{l}9,790.93 \\
9,906.59 \\
9,940.0 \\
9,958.5 \\
9,960.3\end{array}$ & $\begin{array}{l}2 s_{4}-4 p_{1} \\
3 s_{1}^{\prime \prime \prime}-8 \mathrm{~W} \\
3 d_{1}^{\prime}-6 \mathrm{U} \\
3 d_{1}^{\prime \prime}-6 \mathrm{U}\end{array}$ & $\begin{array}{l}9,791.03 \\
9,906.5 \\
9,958.47 \\
9,960.24\end{array}$ \\
\hline
\end{tabular}


TABLE 1.-Infra-red spectrum of neon-Continued

\begin{tabular}{|c|c|c|c|c|}
\hline Intensity & $\lambda_{\mathrm{Bir}}$ I. A. & $\begin{array}{l}\nu_{\mathrm{vac}} \mathrm{cm}^{-1} \\
\text { observed }\end{array}$ & $\begin{array}{l}\text { Term combi- } \\
\text { nation }\end{array}$ & $\begin{array}{l}\nu_{\mathrm{vac}} \mathrm{cm}^{-1} \\
\text { calculated }\end{array}$ \\
\hline $\begin{array}{c}\text { B. S. } \\
4 \\
30 \\
20 \\
1 \\
2\end{array}$ & $\begin{array}{c}\text { B. S. } \\
10,008.55 \\
10,007.31 \\
10,005.54 \\
10,000.9 \\
9,974.2\end{array}$ & $\begin{array}{c}9,988.72 \\
9,989.96 \\
9,991.72 \\
9,996.4 \\
10,023.1\end{array}$ & $\begin{array}{l}3 d_{1}{ }^{\prime}-7 Y \\
3 d_{1}^{\prime}-7 Z \\
3 d_{1}^{\prime \prime}-7 Z \\
3 d_{2}-6 U\end{array}$ & $\begin{array}{r}9,988.68 \\
9,989.94 \\
9,991.71 \\
10,023.27\end{array}$ \\
\hline $\begin{array}{c}6 \\
15 \\
2 \\
7 \mathrm{~h} \\
15\end{array}$ & $\begin{array}{l}9,963.55 \\
9,947.94 \\
9,944.9 \\
9,944.1 \\
9,938.35\end{array}$ & $\begin{array}{l}10,033.84 \\
10,049.58 \\
10,052.6 \\
10,053.5 \\
10,059.28\end{array}$ & $\begin{array}{l}3 s_{1}{ }^{\prime}-7 \mathrm{U} \\
3 s_{1}{ }^{\prime \prime}-7 \mathrm{U} \\
3 d_{3}-6 \mathrm{U} \\
3 d_{2}-7 \mathrm{Y} \\
3 s_{1}^{\prime \prime \prime}-7 \mathrm{U}\end{array}$ & $\begin{array}{l}10,033.82 \\
10,049.54 \\
10,052.68 \\
10,053.48 \\
10,059.30\end{array}$ \\
\hline $\begin{array}{r}10 \\
4 \\
20 \\
30 \\
40\end{array}$ & $\begin{array}{l}9,936.83 \\
9,918.52 \\
9,915.13 \\
9,902.31 \\
9,900.58\end{array}$ & $\begin{array}{l}10,060.81 \\
10,079.39 \\
10,082.83 \\
10,095.89 \\
10,097.64\end{array}$ & $\begin{array}{l}3 s_{1}{ }^{\prime \prime \prime \prime}-7 \mathrm{U} \\
3 d_{3}-7 \mathrm{X} \\
3 d_{3}-7 \mathrm{Y} \\
3 d_{1}^{\prime}-7 \mathrm{~W} \\
3 d_{1}^{\prime \prime}-7 \mathrm{~W}\end{array}$ & $\begin{array}{l}10,060.86 \\
10,079.40 \\
10,082.89 \\
10,095.88 \\
10,097.65\end{array}$ \\
\hline $\begin{array}{r}2 \\
3 \\
2 \\
20 \\
5\end{array}$ & $\begin{array}{l}9,899.06 \\
9,897.30 \\
9,875.90 \\
9,837.47 \\
9,823.42\end{array}$ & $\begin{array}{l}10,099.20 \\
10,101.00 \\
10,122.88 \\
10,162.43 \\
10,176.97\end{array}$ & $\begin{array}{l}3 d_{4}-7 \mathrm{Z} \\
3 d_{4}-7 \mathrm{Z} \\
3 d_{1}^{\prime \prime}-6 p_{5} \\
3 d_{5}-7 \mathrm{X} \\
3 d_{2}-6 p_{1}\end{array}$ & $\begin{array}{l}10,099.21 \\
10,101.04 \\
10,122.7 \\
10,162.37 \\
10,176.90\end{array}$ \\
\hline $\begin{array}{l}1 \\
2 \\
2 \\
1 \\
1\end{array}$ & $\begin{array}{l}9,807.0 \\
9,788.1 \\
9,760.57 \\
9,744.2 \\
9,741.3\end{array}$ & $\begin{array}{l}10,194.0 \\
10,213.7 \\
10,242.50 \\
10,259.7 \\
10,262.9\end{array}$ & $\begin{array}{l}2 s_{2}-5 p_{10} \\
3 d_{6}-7 p_{10} ?\end{array}$ & $\begin{array}{l}10,213.58 \\
10,263.4\end{array}$ \\
\hline $\begin{array}{r}1 \\
1 \\
3 \\
1,000 \\
3\end{array}$ & $\begin{array}{l}9,728.2 \\
9,724.8 \\
9,702.40 \\
9,665.426 \\
9,658.5\end{array}$ & $\begin{array}{l}10,276.6 \\
10,280.2 \\
10,303.90 \\
10,343.33 \\
10,350.7\end{array}$ & $\begin{array}{l}3 s_{1}{ }^{\prime \prime}-9 \mathrm{~W} \\
2 s_{2}-5 p_{8} \\
3 d_{5}-6 p_{4} \\
2 p_{10}-2 s_{5}\end{array}$ & $\begin{array}{l}10,276.74 \\
10,280.16 \\
10,303.8 \\
10,343.34\end{array}$ \\
\hline $\begin{array}{l}1 \\
2 \\
5 \\
3 \\
2\end{array}$ & $\begin{array}{l}9,642.2 \\
9,620.8 \\
9,592.19 \\
9,584.79 \\
9,573.99\end{array}$ & $\begin{array}{l}10,368.2 \\
10,391.3 \\
10,422.29 \\
10,430.34 \\
10,442.10\end{array}$ & $2 s_{2}-5 p_{3}$ & $10,368.18$ \\
\hline $\begin{array}{l}300 \\
500 \\
5 ?+\mathrm{g} \\
3 \\
2\end{array}$ & $\begin{array}{l}9,547.40 \\
9,534.17 \\
9,508.4 \\
9,506.59 \\
9,497.9\end{array}$ & $\begin{array}{l}10,471.18 \\
10,485.71 \\
10,514.1 \\
10,516.14 \\
10,525.8\end{array}$ & $\begin{array}{l}2 p_{2}-3 d_{6} \\
2 p_{2}-3 d_{5} \\
3 d_{1}^{\prime \prime}-8 Z\end{array}$ & $\begin{array}{l}10,471.128 \\
10,485.770 \\
10,513.82\end{array}$ \\
\hline $\begin{array}{c}500 \\
2 \mathrm{~h} \\
300 \\
1 \\
10\end{array}$ & $\begin{array}{l}9,486.68 \\
9,467.8 \\
9,459.21 \\
9,454.0 \\
9,452.08\end{array}$ & $\begin{array}{l}10,538.21 \\
10,559.2 \\
10,568.81 \\
10,574.6 \\
10,576.78\end{array}$ & $\begin{array}{l}2 p_{10}-2 s_{4} \\
3 s_{1}^{\prime}-8 U \\
2 p_{2}-3 d_{3} \\
3 s_{1}^{\prime \prime}-8 U \\
3 d_{2}-8 Z ?\end{array}$ & $\begin{array}{l}10,538.18 \\
10,559.1 \\
10,568.744 \\
10,574.8 \\
10,576.85\end{array}$ \\
\hline $\begin{array}{r}3 \\
2 \\
40 \\
500\end{array}$ & $\begin{array}{l}9,445.26 \\
9,443.8 \\
9,432.94 \\
9,425.38\end{array}$ & $\begin{array}{l}10,584.42 \\
10,586.1 \\
10,598.24 \\
10,606.74\end{array}$ & 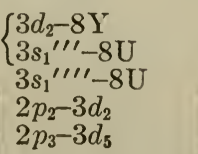 & $\begin{array}{l}10,584.41 \\
10,584.5 \\
10,586.1 \\
10,598.150 \\
10,606.782\end{array}$ \\
\hline
\end{tabular}


TABLE 1.-Infra-red spectrum of neon-Continued

\begin{tabular}{|c|c|c|c|c|}
\hline Intensity & $\lambda_{\text {air }}$ I. A. & $\begin{array}{l}\nu_{\text {vas }} \mathrm{cm}^{-1} \\
\text { observed }\end{array}$ & $\begin{array}{l}\text { Term combi- } \\
\text { nation }\end{array}$ & $\begin{array}{l}\nu_{\mathrm{vac}} \mathrm{cm}^{-1} \\
\text { calculated }\end{array}$ \\
\hline $\begin{array}{c}\text { B. S. } \\
4 \\
6 \\
8 \\
2 \\
5\end{array}$ & $\begin{array}{l}\text { B. S. } \\
9,412.32 \\
9,410.75 \\
9,405.75 \\
9,393.8 \\
9,377.2\end{array}$ & $\begin{array}{l}10,621.46 \\
10,623.23 \\
10,628.88 \\
10,642.4 \\
10,661.2\end{array}$ & $\begin{array}{l}3 d_{4}-8 Z \\
3 d_{4}^{\prime}-8 Z \\
3 d_{4}-8 \mathrm{Y} \\
2 p_{2}-3 d_{1}{ }^{\prime \prime}\end{array}$ & $\begin{array}{l}10,621.32 \\
10,623.15 \\
10,628.88 \\
10,661.187\end{array}$ \\
\hline $\begin{array}{c}200 \\
3 \\
2 \\
1 ? \\
600\end{array}$ & $\begin{array}{l}9,373.28 \\
9,353.3 \\
9,340.5 \\
9,335.0 \\
9,326.52\end{array}$ & $\begin{array}{l}10,665.70 \\
10,688.5 \\
10,703.1 \\
10,709.4 \\
10,719.17\end{array}$ & $\begin{array}{l}2 p_{4}-3 d_{5} \\
3 d_{5}-8 \mathrm{X} \\
3 d_{6}-8 \mathrm{X} \\
3 d_{3}-8 \mathrm{~W} \\
2 p_{3}-3 d_{2}\end{array}$ & $\begin{array}{l}10,665.711 \\
10,688.48 \\
10,703.12 \\
10,709.5 \\
10,719.162\end{array}$ \\
\hline $\begin{array}{r}300 \\
150 \\
600 \\
100 \\
1\end{array}$ & $\begin{array}{l}9,313.98 \\
9,310.58 \\
9,300.85 \\
9,275.53 \\
9,237.4\end{array}$ & $\begin{array}{l}\text { 10, 733. } 61 \\
10,737.52 \\
10,748.76 \\
10,778.10 \\
10,822.6\end{array}$ & $\begin{array}{l}2 p_{4}-3 d_{4} \\
2 p_{5}-3 d_{6} \\
2 p_{4}-3 d_{3} \\
2 p_{4}-3 d_{2}\end{array}$ & $\begin{array}{l}10,733.621 \\
10,737.467 \\
10,748.685 \\
10,778.091\end{array}$ \\
\hline $\begin{array}{l}200 \\
200 \\
400 \\
1 ? \\
2\end{array}$ & $\begin{array}{l}9,226.67 \\
9,221.59 \\
9,220.05 \\
9,214.5 \\
9,212.9\end{array}$ & $\begin{array}{l}10,835.17 \\
10,841.14 \\
10,842.95 \\
10,849.5 \\
10,851.4\end{array}$ & $\begin{array}{l}2 p_{5}-3 d_{3} \\
2 p_{4}-3 d_{1}^{\prime \prime} \\
2 p_{4}-3 d_{1}^{\prime} \\
3 d_{1}^{\prime}-1,378.6 \\
3 d_{1}^{\prime \prime}-1,378.6\end{array}$ & $\begin{array}{l}10,835.083 \\
10,841.128 \\
10,842.893 \\
10,849.5 \\
10,851.3\end{array}$ \\
\hline $\begin{array}{c}600 \\
1 ? \\
3 \\
600 \\
1\end{array}$ & $\begin{array}{l}9,201.76 \\
9,193.8 \\
9,191.8 \\
9,148.68 \\
9,123.2\end{array}$ & $\begin{array}{l}10,864.51 \\
10,873.9 \\
10,876.3 \\
10,927.54 \\
10,958.1\end{array}$ & $\begin{array}{l}2 p_{5}-3 d_{2} \\
3 d_{1}^{\prime \prime}-7 p_{5} \\
\\
2 p_{5}-3 d_{1}^{\prime \prime} \\
3 d_{4}-1,378.6\end{array}$ & $\begin{array}{l}10,864.489 \\
10,874.0 \\
10,927.526 \\
10,958.7\end{array}$ \\
\hline $\begin{array}{l}20 \\
1 ? \\
1 \\
3 \\
1\end{array}$ & $\begin{array}{l}9,121.14 \\
9,115.3 \\
9,111.8 \\
9,103.53 \\
9,102.1\end{array}$ & $\begin{array}{l}10,960.54 \\
10,967.6 \\
10,971.8 \\
10,981.74 \\
10,983.5\end{array}$ & $\begin{array}{l}3 d_{4}^{\prime}-1,378.6 \\
3 d_{3}-9 \mathrm{Y} \\
3 d_{4}-9 \mathrm{Z} \\
3 d_{4}^{\prime}-9 \mathrm{Z}\end{array}$ & $\begin{array}{l}10,960.5 \\
10,967.6 \\
10,981.74 \\
10,983.57\end{array}$ \\
\hline $\begin{array}{l}3 \\
1 \\
1 \\
8 \\
2\end{array}$ & $\begin{array}{l}9,094.9 \\
9,088.5 \\
9,078.2 \\
9,073.04 \\
9,069.7\end{array}$ & $\begin{array}{l}\text { 10,992. } 1 \\
10,999.9 \\
11,012.4 \\
11,018.64 \\
11,022.7\end{array}$ & $2 s_{4}-5 p_{8}$ & $11,018.66$ \\
\hline $\begin{array}{l}1 \\
1 \\
6 \\
3 \\
1\end{array}$ & $\begin{array}{l}9,062.6 \\
9,061.5 \\
9,052.54 \\
9,049.06 \\
9,046.8\end{array}$ & $\begin{array}{l}11,031.3 \\
11,032.7 \\
11,043.60 \\
11,047.84 \\
11,050.6\end{array}$ & $\begin{array}{l}2 s_{4}-5 p_{7} \\
2 s_{4}-5 p_{6} \\
3 d_{5}-9 \mathrm{Y}\end{array}$ & $\begin{array}{l}11,043.52 \\
11,047.88 \\
11,050.6\end{array}$ \\
\hline $\begin{array}{r}2 \\
3 \\
6 \\
200 \\
2\end{array}$ & $\begin{array}{l}9,045.4 \\
9,039.0 \\
9,036.98 \\
8,988.58 \\
8,968.6\end{array}$ & $\begin{array}{l}11,052.3 \\
11,060.1 \\
11,062.61 \\
11,122.18 \\
11,146.9\end{array}$ & $\begin{array}{l}3 d_{2}-9 \mathrm{~W} \\
2 s_{2}-5 p_{4} \\
2 p_{10}-2 s_{3} \\
2 s_{5}-5 p_{10}\end{array}$ & $\begin{array}{l}11,060.09 \\
11,062.72 \\
11,122.18 \\
11,147.02\end{array}$ \\
\hline $\begin{array}{r}3 \\
7 \\
6 \\
10 \\
2\end{array}$ & $\begin{array}{l}8,962.34 \\
8,948.12 \\
8,941.47 \\
8,929.24 \\
8,927.4\end{array}$ & $\begin{array}{l}11,154.74 \\
11,172.47 \\
11,180.78 \\
11,196.09 \\
11,198.4\end{array}$ & $\begin{array}{l}2 s_{2}-5 p_{1} \\
3 d_{5}-9 \mathrm{~W} \\
2 s_{4}-5 p_{3} \\
2 s_{5}-5 p_{9} \\
2 s_{3}-5 p_{2}\end{array}$ & $\begin{array}{l}11,154.83 \\
11,172.47 \\
11,180.82 \\
11,196.03 \\
11,198.49\end{array}$ \\
\hline
\end{tabular}


TABLE 1.-Infra-red spectrum of neon-Continued

\begin{tabular}{|c|c|c|c|c|}
\hline Intensity & $\lambda_{\text {air I. A. }}$ & $\begin{array}{l}\nu_{\mathrm{vac}} \mathrm{cm}^{-1} \\
\text { observed }\end{array}$ & $\begin{array}{l}\text { Term combi- } \\
\text { nation }\end{array}$ & $\begin{array}{l}\nu_{\text {vac }} \mathrm{cm}^{-1} \\
\text { calculated }\end{array}$ \\
\hline $\begin{array}{c}\text { B. S. } \\
300 \\
3 \\
3 \\
2 \\
10\end{array}$ & $\begin{array}{l}\text { B. S. } \\
8,919.50 \\
8,915.44 \\
8,913.0 \\
8,895.6 \\
8,892.22\end{array}$ & $\begin{array}{l}11,208.31 \\
11,213.42 \\
11,216.5 \\
11,238.4 \\
11,242.70\end{array}$ & $\begin{array}{l}2 p_{6}-3 d_{5} \\
2 s_{5}-5 p_{8} \\
2 s_{5}-5 p_{7} \\
2 s_{5}-5 p_{6}\end{array}$ & $\begin{array}{l}11,208.353 \\
11,213.60 \\
11,238.36 \\
11,242.72\end{array}$ \\
\hline $\begin{array}{r}500 \\
100 \\
700 \\
2 \\
50\end{array}$ & $\begin{array}{l}\text { 8, 865. } 76 \\
8,865.33 \\
8,853.88 \\
8,842.1 \\
8,830.92\end{array}$ & $\begin{array}{l}11,276.25 \\
11,276.80 \\
11,291.39 \\
11,306.4 \\
11,320.74\end{array}$ & $\begin{array}{l}2 p_{10}-2 s_{2} \\
2 p_{6}-3 d_{4} \\
2 p_{6}-3 d_{3} \\
2 p_{6}-3 d_{2}\end{array}$ & $\begin{array}{l}11,276.263 \\
11,276.78 \\
11,291.327 \\
11,320.733\end{array}$ \\
\hline $\begin{array}{r}6 \\
30 \\
1,000 \\
50 \\
1,200\end{array}$ & $\begin{array}{l}8,820.36 \\
8,792.51 \\
8,783.78 \\
8,782.01 \\
8,780.65\end{array}$ & $\begin{array}{l}11,334.30 \\
11,370.20 \\
11,381.50 \\
11,383.79 \\
11,385.56\end{array}$ & $\begin{array}{l}2 p_{2}-3 s_{1}{ }^{\prime \prime \prime \prime} \\
2 p_{2}-3 s_{1}^{\prime \prime \prime} \\
2 p_{6}-3 d_{1}^{\prime \prime \prime} \\
2 p_{6}-3 d_{1}^{\prime}\end{array}$ & $\begin{array}{l}11,370.185 \\
11,381.505 \\
11,383.770 \\
11,385.535\end{array}$ \\
\hline $\begin{array}{r}150 \\
400 \\
15 \\
200 \\
500\end{array}$ & $\begin{array}{l}8,778.75 \\
8,771.70 \\
8,767.55 \\
8,704.15 \\
8,681.94\end{array}$ & $\begin{array}{l}11,388.02 \\
11,397.17 \\
11,402.57 \\
11,485.62 \\
11,515.00\end{array}$ & $\begin{array}{l}2 p_{7}-3 d_{6} \\
2 p_{2}-3 s_{1}{ }^{\prime} \\
2 p_{T}-3 d_{5} \\
2 p_{T}-3 d_{3} \\
2 p_{T}-3 d_{2}\end{array}$ & $\begin{array}{l}11,387.977 \\
11,397.226 \\
11,402.619 \\
11,485.593 \\
11,514.999\end{array}$ \\
\hline $\begin{array}{r}500 \\
400 \\
1,500 \\
300 \\
50\end{array}$ & $\begin{array}{l}8,679.51 \\
8,654.51 \\
8,654.380 \\
8,647.05 \\
8,635.31\end{array}$ & $\begin{array}{l}11,518.23 \\
11,550.17 \\
11,551.673 \\
11,561.46 \\
11,577.18\end{array}$ & $\begin{array}{l}2 p_{3}-3 s_{1}{ }^{\prime} \\
2 p_{4}-3 s_{1}{ }^{\prime \prime \prime} \\
2 p_{4}-3 s_{1}{ }^{\prime \prime \prime} \\
2 p_{4}-3 s_{1}{ }^{\prime \prime} \\
2 p_{4}-3 s_{1}{ }^{\prime}\end{array}$ & $\begin{array}{l}11,518.238 \\
11,550.126 \\
11,551.687 \\
11,561.446 \\
11,577.167\end{array}$ \\
\hline $\begin{array}{r}600 \\
400 \\
60 \\
100 \\
60\end{array}$ & $\begin{array}{l}8,634.668 \\
8,591.266 \\
8,582.91 \\
8,571.36 \\
8,544.70\end{array}$ & $\begin{array}{l}11,578.044 \\
11,636.535 \\
11,647.86 \\
11,663.56 \\
11,699.95\end{array}$ & $\begin{array}{l}2 p_{7}-3 d_{1}{ }^{\prime \prime} \\
2 p_{5}-3 s_{1}{ }^{\prime \prime \prime \prime} \\
2 p_{5}-3 s_{1}{ }^{\prime \prime} \\
2 p_{5}-3 s_{1}^{\prime} \\
2 p_{8}-3 d_{5}^{\prime}\end{array}$ & $\begin{array}{l}11,578.036 \\
11,636.524 \\
11,647.844 \\
11,663.565 \\
11,699.996\end{array}$ \\
\hline $\begin{array}{r}500 \\
80 \\
150 \\
400 \\
100\end{array}$ & $\begin{array}{l}8,495.359 \\
8,484.45 \\
8,463.37 \\
8,418.447 \\
8,417.18\end{array}$ & $\begin{array}{l}11,767.904 \\
11,783.03 \\
11,812.38 \\
11,875.416 \\
11,877.20\end{array}$ & $\begin{array}{l}2 p_{8}-3 d_{4} \\
2 p_{8}-3 d_{3} \\
2 p_{8}-3 d_{2} \\
2 p_{8}-3 d_{1}^{\prime \prime} \\
2 p_{8}-3 d_{1}^{\prime}\end{array}$ & $\begin{array}{l}11,767.906 \\
11,782.970 \\
11,812.376 \\
11,875.413 \\
11,877.178\end{array}$ \\
\hline $\begin{array}{l}800 \\
200 \\
150 \\
150 \\
600\end{array}$ & $\begin{array}{l}\text { 8, 377. } 630 \\
8,376.41 \\
8,365.75 \\
8,301.54 \\
\text { 8, 300. 338 }\end{array}$ & $\begin{array}{l}\text { 11, 933. } 274 \\
11,935.02 \\
11,950.22 \\
12,042.65 \\
12,044.396\end{array}$ & $\begin{array}{l}2 p_{9}-3 d_{4}{ }^{\prime} \\
2 p_{9}-3 d_{4} \\
2 p_{9}-3 d_{3} \\
2 p_{9}-3 d_{1}^{\prime \prime} \\
2 p_{9}-3 d_{1}^{\prime}\end{array}$ & $\begin{array}{l}11,933.260 \\
11,935.088 \\
11,950.152 \\
12,042.595 \\
12,044.360\end{array}$ \\
\hline $\begin{array}{r}80 \\
250 \\
150 \\
30 \\
300\end{array}$ & $\begin{array}{l}\text { 8, 267. } 11 \\
\text { 8, 266. } 092 \\
\text { S, 259. } 392 \\
\text { S, 248. 70 } \\
\text { 8, 136. } 423\end{array}$ & $\begin{array}{l}12,092.81 \\
12,094.293 \\
12,104.105 \\
12,119.80 \\
12,287.039\end{array}$ & $\begin{array}{l}2 p_{6}-3 s_{1}{ }^{\prime \prime \prime \prime} \\
2 p_{6}-3 s_{1}{ }^{\prime \prime \prime} \\
2 p_{\sigma}-3 s_{1}^{\prime \prime} \\
2 p_{6}-3 s_{1}^{\prime} \\
2 p_{\tau}-3 s_{1}^{\prime \prime \prime \prime}\end{array}$ & $\begin{array}{l}12,092.768 \\
12,094.329 \\
12,104.088 \\
12,119.809 \\
12,287.034\end{array}$ \\
\hline $\begin{array}{r}60 \\
100 \\
2 \\
200 \\
1\end{array}$ & $\begin{array}{l}\text { S, 128. } 93 \\
8,118.554 \\
\text { 8, 093. } 08 \\
8,182.460 \\
\text { s, 076. } 06\end{array}$ & $\begin{array}{l}12,298.36 \\
12,314.088 \\
12,352.84 \\
12,369.074 \\
12,378.88\end{array}$ & $\begin{array}{l}2 p_{T}-3 s_{1}{ }^{\prime \prime} \\
2 p_{T}-3 s_{1}^{\prime} \\
2 s_{4}-6 p_{3} \\
1 s_{2}-2 p_{10} \\
2 s_{2}-6 p_{1}\end{array}$ & $\begin{array}{l}12,298.354 \\
12,314.075 \\
12,352.85 \\
12,369.077 \\
12,378.92\end{array}$ \\
\hline
\end{tabular}


TABLE 1.-Infra-red spectrum of neon-Continued

\begin{tabular}{|c|c|c|c|c|}
\hline Intensity & $\lambda_{\text {sir }}$ I. A. & $\begin{array}{l}\nu_{\text {rao }} \mathrm{cm}^{-1} \\
\text { observed }\end{array}$ & $\begin{array}{l}\text { Term combi- } \\
\text { nation }\end{array}$ & $\begin{array}{l}\nu_{\mathrm{vac}} \mathrm{cm}^{-1} \\
\text { calculated }\end{array}$ \\
\hline $\begin{array}{c}\text { B. S. } \\
2 \\
2 \\
20 \\
200 \\
70\end{array}$ & $\begin{array}{l}\text { B. S. } \\
8,041.79 \\
8,024.11 \\
7,944.16 \\
7,943.193 \\
7,937.00\end{array}$ & $\begin{array}{l}12,431.63 \\
12,459.02 \\
12,584.41 \\
12,585.938 \\
12,595.76\end{array}$ & $\begin{array}{l}2 s_{5}-6 p_{9} \\
2 s_{5}-6 p_{6} \\
2 p_{8}-3 s_{1}^{\prime \prime \prime \prime} \\
2 p_{8}-3 s_{1}^{\prime \prime \prime} \\
2 p_{8}-3 s_{1}^{\prime \prime}\end{array}$ & $\begin{array}{l}12,431.77 \\
12,459.16 \\
12,584.411 \\
12,585.972 \\
12,595.731\end{array}$ \\
\hline $\begin{array}{r}40 \\
1 \\
30 \\
7 \\
10\end{array}$ & $\begin{array}{l}7,927.13 \\
7,840.04 \\
7,839.08 \\
7,833.06 \\
7,724.63\end{array}$ & $\begin{array}{l}12,611.44 \\
12,751.53 \\
12,753.10 \\
12,762.90 \\
12,942.05\end{array}$ & $\begin{array}{l}2 p_{8}-3 s_{1}{ }^{\prime} \\
2 p_{9}-3 s_{1}{ }^{\prime \prime \prime \prime} \\
2 p_{9}-3 s_{1}{ }^{\prime \prime \prime} \\
2 p_{9}-3 s_{1}{ }^{\prime \prime} \\
2 p_{1}-3 s_{4}\end{array}$ & $\begin{array}{l}12,611.452 \\
12,751.593 \\
12,753.154 \\
12,762.913 \\
12,942.039\end{array}$ \\
\hline $\begin{array}{l}0.5 \\
1 \\
2 \\
0.5 \\
3\end{array}$ & $\begin{array}{l}2,994.410 \\
2,994.250 \\
2,957.293 \\
2,957.148 \\
2,755.82\end{array}$ & $\begin{array}{l}33,385.84 \\
33,387.63 \\
33,804.85 \\
33,806.50 \\
36,276.2\end{array}$ & $\begin{array}{l}1 s_{4}-4 \mathrm{~V} \\
1 s_{4}-4 \mathrm{U} \\
1 s_{5}-4 \mathrm{U} \\
1 s_{5}-4 \mathrm{~T} \\
1 s_{5}-5 \mathrm{U}\end{array}$ & $\begin{array}{l}33,385.9 \\
33,387.5 \\
33,804.9 \\
33,806.5 \\
36,276.2\end{array}$ \\
\hline
\end{tabular}

Wave lengths exceeding $12,066.4 \mathrm{~A}$ are quoted from Hardy's paper, with a correction of $-2.6 \mathrm{~A}$. Analysis of these data showed that a large majority of the 200 infra-red lines observed for neon could be accounted for as combinations of established terms, thus confirming the identity of these terms. About 60 faint lines between 9,100 and $10,900 \mathrm{~A}$ appear to represent combinations of $3 d$ terms with higher members of $f$-type series, and most of these lines have been so classified. Only two terms of this type, $4 x$ and $4 y$ were known heretofore. Hardy states that "the lines in the region around $1.8 \mu$ are apparently due to combinations between the $3 d$ levels and the $x$ and $y$ groups, and although the wave lengths have been checked as well as possible the agreement with the calculated frequencies from the known terms does not check very well with the experimental values." Our interpretation of these lines accounts for only two of them as combinations $3 d-4 x, y$ while the remainder appear to be combinations $3 d-4 z, u, v, w, t$, thus adding five new $f$-type terms. Three of the latter, $4 u, v, t$ are first members of non-Ritzian series and are checked by combinations with $1 s$ terms which represent previously unclassified lines in the ultra-violet, now presented at the end of Table 1. The infra-red data are insufficient to establish any of the $5 f$ terms with certainty, but their approximate values can be obtained from interpolation.

In order to facilitate the comparison of results in Table 1 with the identified spectral terms of neon the latter are collected in Table 2. These data have not been presented in this condensed form heretofore. 


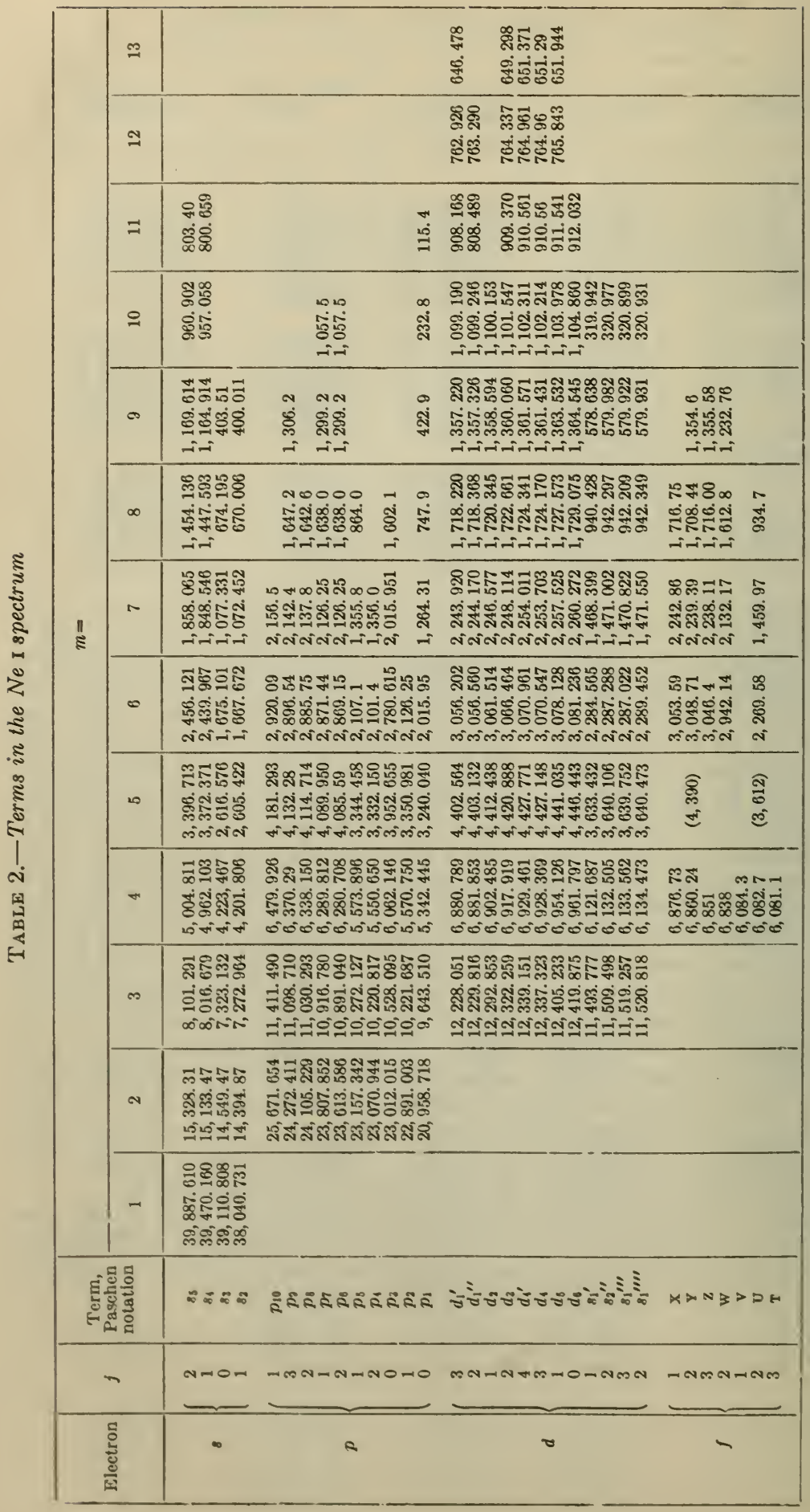




\section{ARGON}

An extensive description and analysis of the first spectrum of argon was published by Meissner ${ }^{14}$ who found the structure of this sprectum very similar to that of neon. Several groups of terms $(2 s, 3 d$, etc.) could not be established because their strongest combinations lay out of range of Meissner's observations in the infra-red. Gremmer ${ }^{15}$ observed 36 new lines in the infra-red, but only 8 of these were explained by Meissner's terms, and 9 of them are not present on our spectrograms.

Important additions to the data for argon were made by Rasmussen ${ }^{16}$ who succeeded, with an efficient spectrograph, an intense light source, and very long exposures to neocyanine plates, in recording infra-red lines to $10,683.4 \mathrm{~A}$. The $2 s$ terms, 5 of the $3 d$ terms, and several additional $f$ terms were identified from these observations.

TABLE 3.-Infra-red spectrum of argon

\begin{tabular}{|c|c|c|c|c|}
\hline Intensity & $\lambda_{\text {air }}$ I. A. & $\begin{array}{l}\nu_{\text {vao }} \mathrm{cm}^{-1} \\
\text { observed }\end{array}$ & $\begin{array}{l}\text { Term combi- } \\
\text { nation }\end{array}$ & $\begin{array}{l}\nu_{\mathrm{Vac}} \mathrm{cm}^{-1} \\
\text { calculated }\end{array}$ \\
\hline $\begin{array}{r}\text { Paschen } \\
4 \\
4 \\
31\end{array}$ & $\begin{array}{l}\text { Paschen } \\
13,719.9 \\
13,504.6 \\
12,499\end{array}$ & $\begin{array}{l}7,287.3 \\
7,402.9 \\
7,998.5\end{array}$ & $\begin{array}{l}2 p_{9}-3 d_{4}{ }^{\prime} \\
2 p_{8}-3 d_{4} \\
2 p_{9}-2 s_{5}\end{array}$ & $\begin{array}{l}7,287.60 \\
7,403.12 \\
8,005.65\end{array}$ \\
\hline B. S. $\begin{array}{r}1 \\
2\end{array}$ & $\begin{array}{c}\text { B. S. } \\
11,720.0 \\
11,668.9\end{array}$ & $\begin{array}{l}8,530.1 \\
8,567.44\end{array}$ & $\begin{array}{l}2 p_{8}-3 d_{2} \\
2 p_{6}-3 s_{1}{ }^{\prime \prime}\end{array}$ & $\begin{array}{l}8,530.29 \\
8,567.56\end{array}$ \\
\hline $\begin{array}{l}5 \\
2 \\
1 \\
2 \\
5\end{array}$ & $\begin{array}{l}11,488.1 \\
11,441.7 \\
11,393.53 \\
11,106.41 \\
11,078.87\end{array}$ & $\begin{array}{l}8,702.31 \\
8,737.59 \\
8,774.52 \\
9,001.35 \\
9,023.72\end{array}$ & $\begin{array}{l}1 s_{2}-2 p_{10} \\
2 p_{6}-2 s_{2} \\
2 p_{7}-2 s_{3} \\
3 d_{1}-5 \mathrm{U} \\
2 p_{8}-3 s_{1}^{\prime \prime \prime \prime}\end{array}$ & $\begin{array}{l}8,702.20 \\
8,737.50 \\
8,774.30 \\
9,001.19 \\
9,023.67\end{array}$ \\
\hline $\begin{array}{r}5 \\
2 \\
10 \\
1 \\
4\end{array}$ & $\begin{array}{l}10,950.73 \\
10,892.39 \\
10,880.92 \\
10,860.95 \\
10,773.33\end{array}$ & $\begin{array}{l}9,129.31 \\
9,178.21 \\
9,187.88 \\
9,204.78 \\
9,279.64\end{array}$ & $\begin{array}{l}2 p_{6}-3 s_{1}{ }^{\prime} \\
2 p_{9}-3 s_{1}{ }^{\prime \prime \prime \prime} \\
2 p_{8}-3 s_{1}^{\prime \prime} \\
2 p_{8}-3 s_{1}^{\prime \prime \prime} \\
2 p_{T}-3 s_{1}^{\prime}\end{array}$ & $\begin{array}{l}9,129.31 \\
9,178.24 \\
9,187.78 \\
9,204.69 \\
9,279.60\end{array}$ \\
\hline $\begin{array}{l}2 \\
6 \\
5 \\
1 \\
4\end{array}$ & $\begin{array}{l}10,770.33 \\
10,759.12 \\
10,733.88 \\
10,722.1 \\
10,712.76\end{array}$ & $\begin{array}{l}9,282.22 \\
9,291.90 \\
9,313.74 \\
9,323.98 \\
9,332.11\end{array}$ & $\begin{array}{l}3 d_{4}^{\prime \prime}-5 \mathrm{Y} \\
3 d_{4}^{\prime \prime}-5 \mathrm{U} \\
3 s_{1}^{\prime \prime \prime}-5 \mathrm{~W} \\
2 p_{10}-3 d_{1}^{\prime \prime} \\
3 s_{1}^{\prime \prime}-5 Z\end{array}$ & $\begin{array}{l}9,282.17 \\
9,291.97 \\
9,313.81 \\
9,324.00 \\
9,332.13\end{array}$ \\
\hline $\begin{array}{r}8 \\
3 \\
40 \\
100 \\
10\end{array}$ & $\begin{array}{l}10,700.97 \\
10,683.40 \\
10,681.80 \\
10,673.61 \\
10,529.31\end{array}$ & $\begin{array}{l}9,342.39 \\
9,357.76 \\
9,359.16 \\
9,366.35 \\
9,494.70\end{array}$ & $\begin{array}{l}2 p_{9}-3 s_{1}{ }^{\prime \prime} \\
2 p_{8}-2 s_{2} \\
2 p_{9}-3 s_{1}{ }^{\prime \prime \prime} \\
2 p_{10}-2 s_{5} \\
3 s_{1}{ }^{\prime \prime \prime \prime}-5 \mathrm{~W}\end{array}$ & $\begin{array}{l}9,342.35 \\
9,357.72 \\
9,359.36 \\
9,366.37 \\
9,494.83\end{array}$ \\
\hline $\begin{array}{r}30 \\
100 \\
300 \\
1 \\
60\end{array}$ & $\begin{array}{l}10,506.48 \\
10,478.09 \\
10,470.09 \\
10,357.6 \\
10,332.76\end{array}$ & $\begin{array}{l}9,515.33 \\
9,541.11 \\
9,548.41 \\
9,652.1 \\
9,675.31\end{array}$ & $\begin{array}{l}3 d_{3}-4 Z \\
2 p_{10}-2 s_{4} \\
1 s_{5}-2 p_{10} \\
3 d_{5}-4 p_{5} \\
3 d_{4}-5 \mathrm{~V}\end{array}$ & $\begin{array}{l}9,515.46 \\
9,541.11 \\
9,548.36 \\
9,652.27 \\
9,675.34\end{array}$ \\
\hline
\end{tabular}

14 K. W. Meissner, Zeitschr. 1. Phys., vol. 37, p. 238, 1926; vol. 39, p. 172, 1926; vol. 40, p. 839, 1927. $15 \mathrm{~W}$. Gremmer, Zeitschr. f. Phys., vol. 50, p. 721, 1928.

16 E. Rasmussen, Zeitschr. 1. Phys., vol. 75, p. 695, 1932. 
TABLE 3.-Infra-red spectrum of argon-Continued

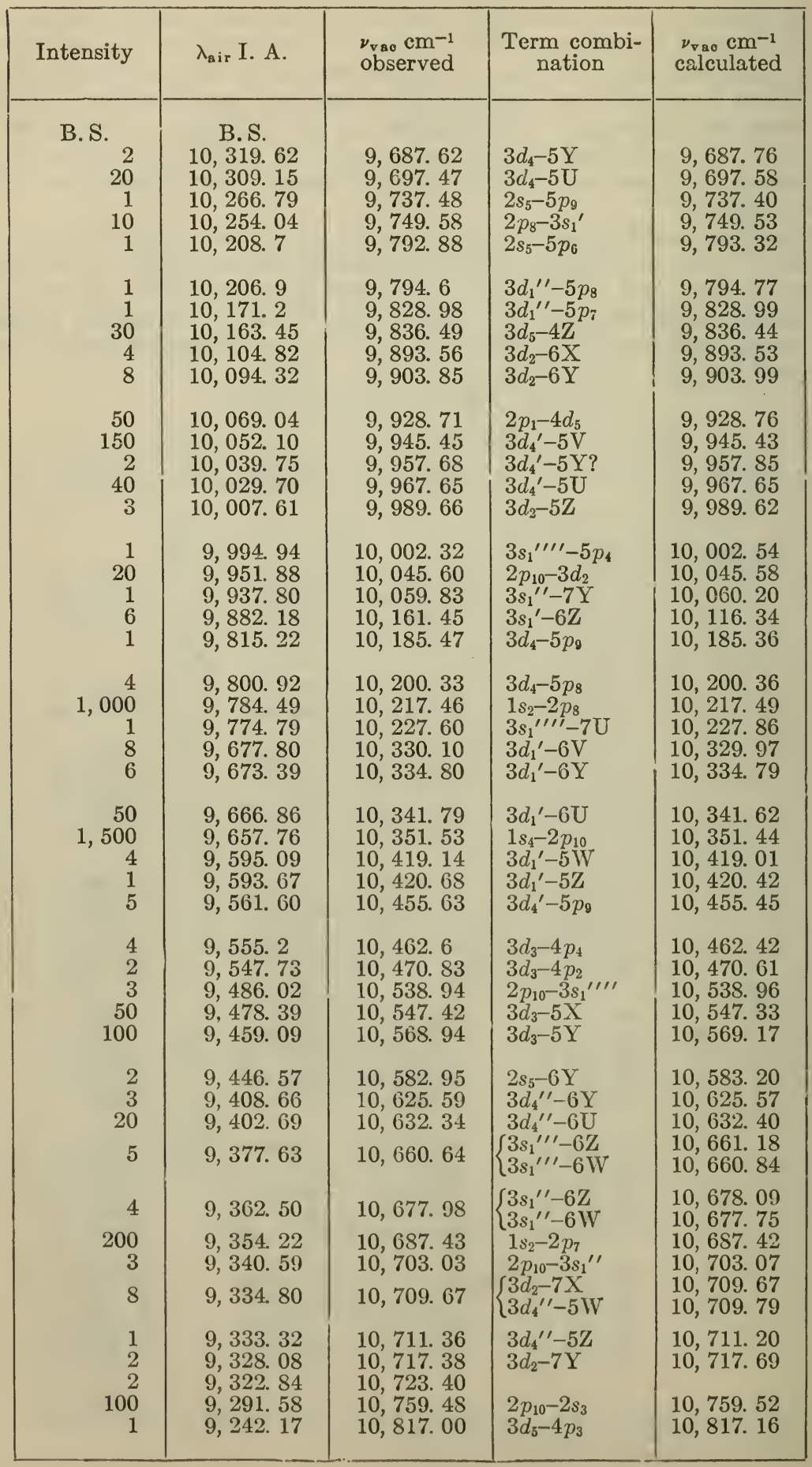


TABLE 3.-Infra-red spectrum of argon-Continued

\begin{tabular}{|c|c|c|c|c|}
\hline Intensity & $\lambda_{\text {air I. A. }}$ & $\begin{array}{l}\nu_{\mathrm{Vac}} \mathrm{cm}^{-1} \\
\text { observed }\end{array}$ & $\begin{array}{l}\text { Term combi- } \\
\text { nation }\end{array}$ & $\begin{array}{c}\nu_{\mathrm{rac}} \mathrm{cm}^{-1} \\
\text { calculated }\end{array}$ \\
\hline $\begin{array}{c}\text { B. S, } \\
1,000 \\
5 \text { h } \\
50 \\
150 \\
6\end{array}$ & $\begin{array}{c}\text { B. S. } \\
9,224.49 \\
9,221.08 \\
9,198.61 \\
9,194.68 \\
9,180.17\end{array}$ & $\begin{array}{l}10,837.73 \\
10,841.74 \\
10,868.23 \\
10,872.87 \\
10,890.06\end{array}$ & $\begin{array}{l}1 s_{2}-2 p_{6} \\
3 s_{1}{ }^{\prime \prime \prime \prime}-6 \mathrm{~W} \\
3 d_{5}-5 \mathrm{X} \\
2 p_{10}-2 s_{2} \\
3 d_{5}-5 \mathrm{Y}\end{array}$ & $\begin{array}{l}10,837.71 \\
10,841.86 \\
10,868.31 \\
10,873.01 \\
10,890.15\end{array}$ \\
\hline $\begin{array}{r}500 \\
1 \\
60 \\
50 \\
40\end{array}$ & $\begin{array}{l}9,122.95 \\
9,111.3 \\
9,075.42 \\
9,073.34 \\
9,066.77\end{array}$ & $\begin{array}{l}10,958.36 \\
10,972.4 \\
11,015.75 \\
11,018.28 \\
11,026.26\end{array}$ & $\begin{array}{l}1 s_{5}-2 p_{10} \\
3 d_{5}-4 p_{1} \\
2 p_{2}-4 d_{6} \\
3 d_{6}-5 \mathrm{X} \\
3 d_{4}-6 \mathrm{~V}\end{array}$ & $\begin{array}{l}10,958.28 \\
10,972.54 \\
11,015.64 \\
11,018.44 \\
11,026.34\end{array}$ \\
\hline $\begin{array}{c}2 \\
4 \mathrm{~h} \\
10 \\
1 \\
3\end{array}$ & $\begin{array}{l}9,057.51 \\
9,057.23 \\
8,994.09 \\
8,992.84 \\
8,988.20\end{array}$ & $\begin{array}{l}11,037.53 \\
11,037.88 \\
11,115.36 \\
11,116.91 \\
11,122.65\end{array}$ & $\begin{array}{l}2 p_{1}-3 s_{4} \\
3 d_{4}-6 \mathrm{U} \\
3 d_{4}-5 \mathrm{~W} \\
3 d_{4}-5 \mathrm{Z} \\
3 d_{3}-5 p_{6}\end{array}$ & $\begin{array}{l}11,037.54 \\
11,037.99 \\
11,115.38 \\
11,116.79 \\
11,122.69\end{array}$ \\
\hline $\begin{array}{r}2 \\
2 \\
10 \\
40 \\
1\end{array}$ & $\begin{array}{l}\text { 8, 970. } 98 \\
8,967.39 \\
8,964.48 \\
8,962.19 \\
8,895.42\end{array}$ & $\begin{array}{l}11,144.00 \\
11,148.46 \\
11,152.08 \\
11,154.93 \\
11,238.66\end{array}$ & $\begin{array}{l}3 d_{1}^{\prime}-7 \mathrm{~V} \\
3 d_{1}^{\prime}-7 \mathrm{Y} \\
3 d_{1}^{\prime}-7 \mathrm{U} \\
2 p_{2}-4 d_{5} \\
3 d_{2}-8 \mathrm{X}\end{array}$ & $\begin{array}{l}11,143.96 \\
11,148.49 \\
11,152.04 \\
11,154.97 \\
11,238.81\end{array}$ \\
\hline $\begin{array}{r}1 \\
4 \\
150 \\
1 \\
20\end{array}$ & $\begin{array}{l}\text { 8, 891. } 70 \\
8,874.84 \\
8,849.97 \\
8,846.17 \\
8,840.82\end{array}$ & $\begin{array}{l}11,243.36 \\
11,264.72 \\
11,296.37 \\
11,301.23 \\
11,308.07\end{array}$ & $\begin{array}{l}3 d_{2}-8 \mathrm{Y} \\
2 p_{10}-3 s_{1}{ }^{\prime} \\
3 d_{4}{ }^{\prime}-6 \mathrm{~V} \\
3 d_{4}{ }^{\prime}-6 \mathrm{Y} \\
3 d_{6}-6 \mathrm{U}\end{array}$ & $\begin{array}{l}11,243.94 \\
11,264.82 \\
11,296.43 \\
11,301.25 \\
11,308.08\end{array}$ \\
\hline $\begin{array}{r}3 \\
1 \\
3 \\
100 \\
30\end{array}$ & $\begin{array}{l}8,840.39 \\
8,819.37 \\
8,805.16 \\
8,799.13 \\
8,784.59\end{array}$ & $\begin{array}{l}11,308.61 \\
11,335.57 \\
11,353.86 \\
11,361.64 \\
11,380.44\end{array}$ & $\begin{array}{l}3 d_{2}-6 \mathrm{Z} \\
3 d_{5}-5 p_{10} ? \\
2 p_{3}-4 d_{5} \\
2 p_{4}-4 d_{6}\end{array}$ & $\begin{array}{l}11,335.58 \\
11,354.45 \\
11,361.69 \\
11,380.35\end{array}$ \\
\hline $\begin{array}{r}200 \\
1 \\
3 \\
20 \\
2\end{array}$ & $\begin{array}{l}8,761.72 \\
8,741.26 \\
8,739.51 \\
8,736.63 \\
8,736.19\end{array}$ & $\begin{array}{l}11,410.12 \\
11,436.86 \\
11,439.15 \\
11,442.92 \\
11,443.50\end{array}$ & $\begin{array}{l}2 p_{2}-4 d_{3} \\
3 d_{5}-5 p_{7} \\
3 d_{1}^{\prime \prime}-7 \mathrm{Y} \\
3 d_{1}^{\prime \prime}-7 \mathrm{U} \\
3 d_{5}-5 p_{6}\end{array}$ & $\begin{array}{l}11,410.19 \\
11,436.97 \\
11,439.27 \\
11,442.82 \\
11,443.67\end{array}$ \\
\hline $\begin{array}{r}5 \\
3 \\
2 \\
60 \\
400\end{array}$ & $\begin{array}{l}8,713.79 \\
8,700.95 \\
8,690.12 \\
8,678.43 \\
8,667.94\end{array}$ & $\begin{array}{l}11,472.91 \\
11,489.84 \\
11,504.16 \\
11,519.66 \\
11,533.60\end{array}$ & $\begin{array}{l}3 s_{1}{ }^{\prime \prime \prime}-7 Z \\
3 s_{1}{ }^{\prime \prime}-7 Z \\
3 d_{6}-5 p_{10} \\
2 p_{4}-4 d_{5} \\
1 s_{3}-2 p_{7}\end{array}$ & $\begin{array}{l}11,472.92 \\
11,489.83 \\
11,504.55 \\
11,519.68 \\
11,533.58\end{array}$ \\
\hline $\begin{array}{r}1 \\
100 \\
150 \\
4 \\
5\end{array}$ & $\begin{array}{l}8,642.89 \\
8,620.47 \\
8,605.78 \\
8,579.49 \\
8,578.06\end{array}$ & $\begin{array}{l}11,567.03 \\
11,597.11 \\
11,616.91 \\
11,652.51 \\
11,654.45\end{array}$ & $\begin{array}{l}3 d_{5}-5 p_{5} \\
2 p_{5}-4 d_{5} \\
2 p_{3}-4 d_{3} \\
3 s_{s^{\prime \prime \prime \prime}}-7 \mathrm{~W} \\
1 s_{2}-2 p_{5}\end{array}$ & $\begin{array}{l}11,567.10 \\
11,597.10 \\
11,616.91 \\
11,652.5 \\
11,654.45\end{array}$ \\
\hline
\end{tabular}


TABLE 3.-Infra-red spectrum of argon-Continued

\begin{tabular}{|c|c|c|c|c|}
\hline Intensity & $\lambda_{\text {air }}$ I. A. & $\begin{array}{l}\nu_{\mathrm{vac}} \mathrm{cm}^{-1} \\
\text { observed }\end{array}$ & $\begin{array}{l}\text { Term combi- } \\
\text { nation }\end{array}$ & $\begin{array}{l}\nu_{\mathrm{Vac}} \mathrm{cm}^{-1} \\
\text { calculated }\end{array}$ \\
\hline $\begin{array}{r}\text { B.S. } \\
1 \\
1 \\
3 \\
2,000 \\
2\end{array}$ & $\begin{array}{l}\text { B. S. } \\
8,565.13 \\
8,563.38 \\
8,561.38 \\
8,521.442 \\
8,496.64\end{array}$ & $\begin{array}{l}11,672.04 \\
11,674.43 \\
11,677.16 \\
11,731.884 \\
11,766.13\end{array}$ & $\begin{array}{l}3 d_{1}^{\prime}-8 \mathrm{~V} \\
3 d_{1}^{\prime}-8 \mathrm{Y} \\
3 d_{1}^{\prime}-8 U \\
1 s_{2}-2 p_{4} \\
\left\{\begin{array}{l}3 d_{1}^{\prime}-6 Z \\
3 d_{1}^{\prime}-6 \mathrm{~W}\end{array}\right.\end{array}$ & $\begin{array}{l}11,672.00 \\
11,674.74 \\
11,677.09 \\
11,731.87 \\
11,766.38 \\
11,766.04\end{array}$ \\
\hline $\begin{array}{r}40 \\
20 \\
1 \\
6 \\
2,500\end{array}$ & $\begin{array}{l}8,490.30 \\
8,443.44 \\
8,440.26 \\
8,437.71 \\
8,424.648\end{array}$ & $\begin{array}{l}11,774.92 \\
11,840.27 \\
11,844.73 \\
11,848.30 \\
11,866.675\end{array}$ & $\begin{array}{l}2 p_{4}-4 d_{3} \\
3 d_{4}-7 \mathrm{~V} \\
3 d_{4}-7 \mathrm{Y} \\
3 d_{4}-7 \mathrm{U} \\
1 s_{4}-2 p_{8}\end{array}$ & $\begin{array}{l}11,744.90 \\
11,840.33 \\
11,844.86 \\
11,848.41 \\
11,866.73\end{array}$ \\
\hline $\begin{array}{r}3,000 \\
20 \\
80 \\
60 \\
3\end{array}$ & $\begin{array}{l}8,408.213 \\
8,399.35 \\
8,392.28 \\
8,384.73 \\
8,367.03\end{array}$ & $\begin{array}{l}11,889.870 \\
11,902.42 \\
11,912.44 \\
11,923.17 \\
11,948.39\end{array}$ & $\begin{array}{l}1 s_{2}-2 p_{3} \\
3 d_{3}-6 \mathrm{X} \\
3 d_{3}-6 \mathrm{Y} \\
2 p_{3}-4 d_{4} \\
2 p_{2}-4 d_{1}^{\prime \prime}\end{array}$ & $\begin{array}{l}11,889.86 \\
11,902.11 \\
11,912.57 \\
11,923.17 \\
11,948.49\end{array}$ \\
\hline $\begin{array}{r}1 \\
4 \\
20 \\
1 \\
1\end{array}$ & $\begin{array}{l}8,355.30 \\
8,353.50 \\
8,332.21 \\
8,305.02 \\
8,303.79\end{array}$ & $\begin{array}{l}22,965.17 \\
11,967.75 \\
11,998.32 \\
12,037.60 \\
12,039.39\end{array}$ & $\begin{array}{l}3 d_{1}^{\prime \prime}-8 \mathrm{Y} \\
3 d_{1}^{\prime \prime}-8 \mathrm{U} \\
3 d_{3}-5 \mathrm{Z} \\
3 d_{1}^{\prime}-9 \mathrm{Y} \\
3 d_{1}^{\prime}-9 \mathrm{U}\end{array}$ & $\begin{array}{l}11,965.52 \\
11,967.87 \\
11,998.20 \\
12,037.6 \\
12,039.39\end{array}$ \\
\hline $\begin{array}{r}8 \\
1,500 \\
50 \\
4 \\
6\end{array}$ & $\begin{array}{l}8,291.88 \\
8,264.524 \\
8,255.07 \\
8,249.58 \\
8,224.72\end{array}$ & $\begin{array}{l}12,056.68 \\
12,096.589 \\
12,110.44 \\
12,118.50 \\
12,155.13\end{array}$ & $\begin{array}{l}3 d_{4}{ }^{\prime \prime}-6 \mathrm{~W} \\
1 s_{2}-2 p_{2} \\
3 d_{4}^{\prime}-7 \mathrm{~V} \\
3 d_{4}^{\prime}-7 \mathrm{U} \\
2 p_{3}-4 d_{1}^{\prime \prime}\end{array}$ & $\begin{array}{l}12,056.82 \\
12,096.58 \\
12,110.42 \\
12,118.50 \\
12,155.21\end{array}$ \\
\hline $\begin{array}{r}20 \\
20 \\
40 \\
10 \\
2\end{array}$ & $\begin{array}{l}8,203.42 \\
8,178.96 \\
8,178.84 \\
8,171.95 \\
8,166.21\end{array}$ & $\begin{array}{l}12,186.69 \\
12,223.14 \\
12,223.32 \\
12,233.62 \\
12,242.22\end{array}$ & $\begin{array}{l}2 p_{2}-3 s_{5} \\
3 d_{5}-6 \mathrm{X} \\
3 d_{5}-6 \mathrm{Y}\end{array}$ & $\begin{array}{l}12,186.74 \\
12,223.09 \\
12,233.55\end{array}$ \\
\hline $\begin{array}{r}3 \\
10 \\
2 \\
50 \\
4,000\end{array}$ & $\begin{array}{l}8,151.86 \\
8,143.50 \\
8,127.30 \\
8,119.18 \\
8,115.309\end{array}$ & $\begin{array}{l}12,263.77 \\
12,276.36 \\
12,300.83 \\
12,313.13 \\
12,319.006\end{array}$ & $\begin{array}{l}2 p_{2}-3 s_{4} \\
2 p_{3}-4 d_{1}^{\prime} \\
\\
2 p_{4}-4 d_{1}^{\prime \prime} \\
1 s_{5}-2 p_{9}\end{array}$ & $\begin{array}{l}12,263.75 \\
12,276.36 \\
12,313.20 \\
12,319.00\end{array}$ \\
\hline $\begin{array}{r}2,000 \\
20 \\
5 \\
20\end{array}$ & $\begin{array}{l}8,103.692 \\
8,094.06 \\
8,082.93 \\
8,079.68 \\
8,066.60\end{array}$ & $\begin{array}{l}12,336.665 \\
12,351.35 \\
12,368.35 \\
12,373.32 \\
12,393.39\end{array}$ & $\begin{array}{r}1 s_{4}-2 p_{7} \\
2 p_{2}-4 d_{2} \\
3 d_{4}-8 \mathrm{~V} \\
\left\{\begin{array}{l}3 d_{6}-6 \mathrm{X} \\
3 d_{4}-8 \mathrm{U} \\
2 p_{3}-3 s_{5}\end{array}\right.\end{array}$ & $\begin{array}{l}12,336.66 \\
12,351.34 \\
12,368.36 \\
12,373.19 \\
12,373.46 \\
12,393.46\end{array}$ \\
\hline $\begin{array}{r}1 \\
100 \\
50 \\
20 \\
2\end{array}$ & $\begin{array}{l}8,058.53 \\
8,053.32 \\
8,046.13 \\
8,037.23 \\
8,021.9\end{array}$ & $\begin{array}{l}12,405.80 \\
12,413.83 \\
12,424.92 \\
12,438.68 \\
12,462.4\end{array}$ & $\begin{array}{l}2 p_{6}-4 d_{5} \\
2 p_{7}-4 d_{4} \\
2 p_{1}-3 s_{2} \\
3 d_{4}-6 \mathrm{~W}\end{array}$ & $\begin{array}{l}12,413.84 \\
12,424.80 \\
12,438.70 \\
12,462.41\end{array}$ \\
\hline
\end{tabular}


TABLE 3.-Infra-red spectrum of argon-Continued

\begin{tabular}{|c|c|c|c|c|}
\hline Intensity & $\lambda_{\mathrm{air}}$ I.YA. & $\begin{array}{l}\nu_{\text {vao }} \mathrm{cm}^{-1} \\
\text { observed }\end{array}$ & $\begin{array}{l}\text { Term combi- } \\
\text { nation }\end{array}$ & $\begin{array}{l}\nu_{\mathrm{Vac}} \mathrm{cm}^{-1} \\
\text { calculated }\end{array}$ \\
\hline $\begin{array}{r}\text { B. S. } \\
800 \\
600 \\
3 \\
2 \\
10\end{array}$ & $\begin{array}{l}\text { B. S. } \\
8,014.785 \\
8,006.157 \\
7,965.08 \\
7,960.84 \\
7,956.99\end{array}$ & $\begin{array}{l}12,473.515 \\
12,486.957 \\
12,551.35 \\
12,558.04 \\
12,564.12\end{array}$ & $\begin{array}{l}1 s_{5}-2 p_{8} \\
1 s_{4}-2 p_{6} \\
2 p_{4}-3 s_{5} \\
2 p_{3}-4 d_{2} \\
2 p_{T}-4 d_{5}\end{array}$ & $\begin{array}{l}12,473.57 \\
12,486.95 \\
12,551.45 \\
12,558.06 \\
12,564.13\end{array}$ \\
\hline $\begin{array}{r}400 \\
20 \\
4 \\
1 \\
100\end{array}$ & $\begin{array}{l}7,948.176 \\
7,916.45 \\
7,910.23 \\
7,906.91 \\
7,891.10\end{array}$ & $\begin{array}{l}12,578.048 \\
12,628.45 \\
12,638.40 \\
12,643.69 \\
12,669.02\end{array}$ & $\begin{array}{l}1 s_{3}-2 p_{4} \\
2 p_{4}-3 s_{4} \\
3 d_{4}^{\prime}-8 \mathrm{~V} \\
3 d_{4}^{\prime}-8 \mathrm{U} \\
2 p_{6}-4 d_{3}\end{array}$ & $\begin{array}{l}12,578.03 \\
12,628.46 \\
12,638.35 \\
12,643.55 \\
12,669.06\end{array}$ \\
\hline $\begin{array}{r}40 \\
15 \\
2 \\
8 \\
1\end{array}$ & $\begin{array}{l}7,868.20 \\
7,861.91 \\
7,860.44 \\
7,855.73 \\
7,853.29\end{array}$ & $\begin{array}{l}12,705.90 \\
12,716.06 \\
12,718.44 \\
12,726.06 \\
12,730.02\end{array}$ & $\begin{array}{l}2 p_{5}-3 s_{4} \\
2 p_{4}-4 d_{2} \\
3 d_{3}-7 \mathrm{X} \\
3 d_{3}-7 \mathrm{Y} \\
3 d_{4}-9 \mathrm{~V}\end{array}$ & $\begin{array}{l}12,705.88 \\
12,716.05 \\
12,718.26 \\
12,726.27 \\
12,730.04\end{array}$ \\
\hline $\begin{array}{r}10 \\
30 \\
200 \\
200\end{array}$ & $\begin{array}{l}7,814.33 \\
7,798.55 \\
7,724.210 \\
7,723.759\end{array}$ & $\begin{array}{l}12,793.49 \\
12,819.37 \\
12,942.749 \\
12,943.507\end{array}$ & $\begin{array}{l}2 p_{5}-4 d_{2} \\
2 p_{7}-4 d_{3} \\
1 s_{3}-2 p_{2} \\
1 s_{5}-2 p_{7}\end{array}$ & $\begin{array}{l}12,793.47 \\
12,819.35 \\
12,942.74 \\
12,943.50\end{array}$ \\
\hline $\begin{array}{r}20 \\
2 \\
50 \\
4\end{array}$ & $\begin{array}{l}7,704.81 \\
7,690.10 \\
7,670.04 \\
7,667.03\end{array}$ & $\begin{array}{l}12,975.34 \\
13,000.16 \\
13,034.16 \\
13,039.28\end{array}$ & $\begin{array}{l}2 p_{6}-4 d_{4} \\
3 d_{4}{ }^{\prime}-9 \mathrm{~V} \\
2 p_{8}-4 d_{5} \\
3 d_{5}-7 \mathrm{X}\end{array}$ & $\begin{array}{l}12,975.32 \\
13,000.13 \\
13,034.06 \\
13,039.24\end{array}$ \\
\hline $\begin{array}{r}2 \\
500 \\
50 \\
30\end{array}$ & $\begin{array}{l}7,662.3 \\
7,635.107 \\
7,628.86 \\
7,618.33\end{array}$ & $\begin{array}{l}13,047.3 \\
13,093.795 \\
13,104.52 \\
13,122.63\end{array}$ & $\begin{array}{l}3 d_{5}-7 \mathrm{Y} \\
1 s_{5}-2 p_{6} \\
2 p_{2}-4 s_{1}^{\prime \prime \prime \prime} \\
2 p_{2}-4 s_{1}^{\prime \prime}\end{array}$ & $\begin{array}{l}13,047.25 \\
13,093.79 \\
13,104.44 \\
13,122.58\end{array}$ \\
\hline
\end{tabular}

Our results for the infra-red lines in the first spectrum of argon are displayed in Table 3 , which is constructed exactly like Table 1. Analysis of these new data has yielded improved values for the four $2 s$ terms, has identified all 12 of the $3 d$ terms, and extended the $f$-type sequences, so that the recognition of energy states for neutral argon may now be regarded as complete. The $2 s$ terms were evaluated from combinations with Meissner's $2 p$ terms, the $d$ terms from Meissner's $f$ and $p$ terms, and the new $f$ terms were based upon combinations with the derived $d$ terms. All of the known spectral terms of the $\Lambda$ I spectrum are collected in Table 4, which is similar to Table 2.

Radiometric investigation of this spectrum should reveal many intense lines which may be expected from combinations of known terms, but which lie beyond the present limit of photographic detection. Three such lines were observed by Paschen ${ }^{17}$ many years ago, their wave lengths (corrected to the I. A. scale) and probable classifications appear at the head of Table 3.

17 F. Paschen, Ann. d. Phys. (4), vol. 27, p. 537, 1908.

$161541-33-2$ 


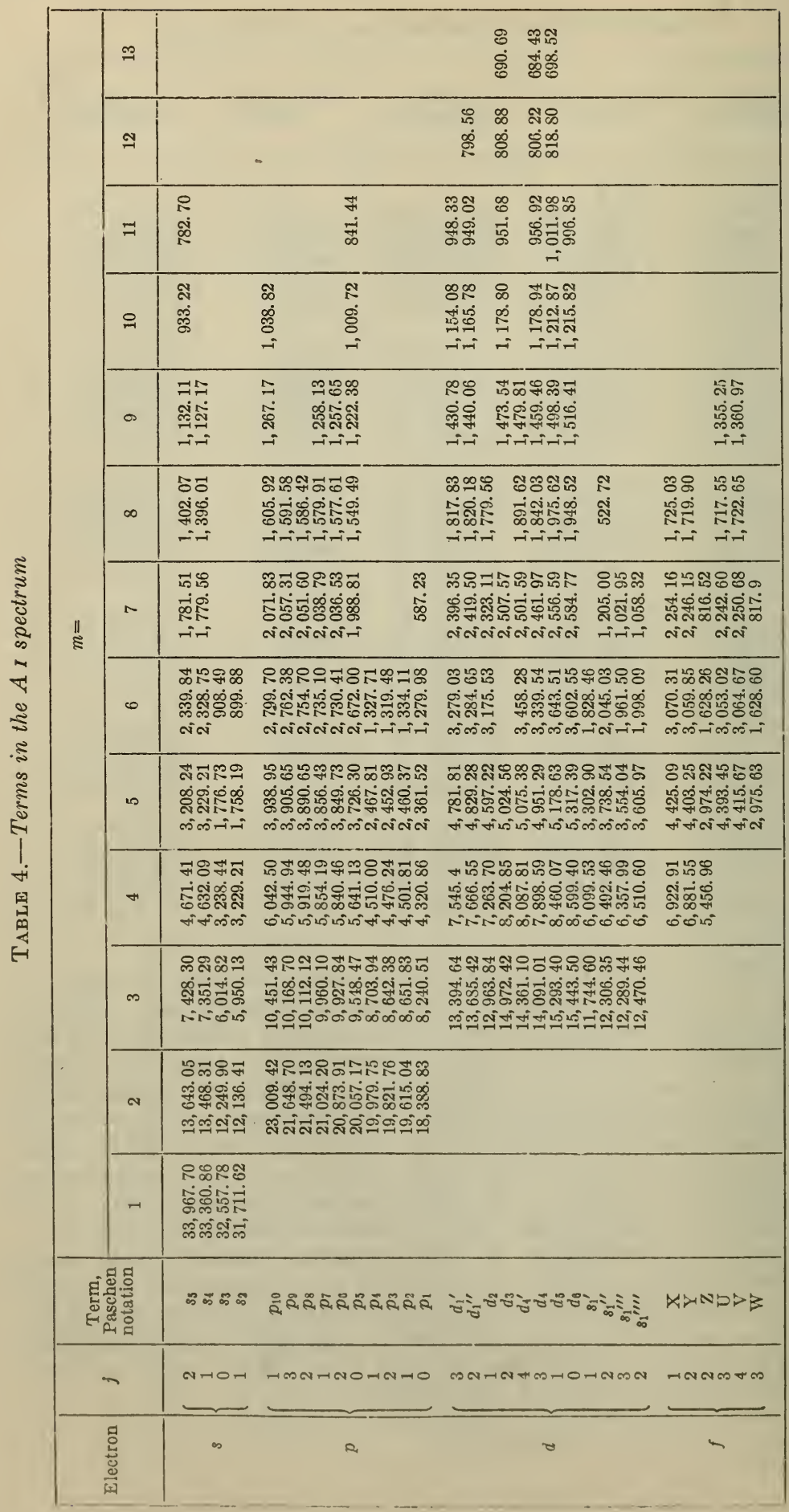




\section{KRYPTON}

The first spectrum of krypton was analyzed simultaneously by Gremmer ${ }^{18}$ and by Meggers, de Bruin, and Humphreys. ${ }^{19}$ Our preliminary description and analysis was extended 20 two years later, when the number of observed and classified lines was doubled. Gremmer ${ }^{21}$ then reported his own analysis to be in perfect agreement with ours if a revised interpretation be given to six of our levels. Up to this time eight of the $3 d$ terms were still lacking. With long exposures Rasmussen ${ }^{22}$ recorded additional lines mainly in the red and infra-red which led to the identification of five of the missing $3 d$ terms, an extension of the terms and a few minor changes among the higher terms.

TABLE 5.-Infra-red spectrum of krypton

\begin{tabular}{|c|c|c|c|c|}
\hline Intensity & $\lambda_{\text {a ir I. A. }}$ & $\begin{array}{l}\nu_{\text {vac }} \mathrm{cm}^{-1} \\
\text { observed }\end{array}$ & $\begin{array}{l}\text { Term combi- } \\
\text { nation }\end{array}$ & $\begin{array}{l}\nu_{\text {vao }} \mathrm{cm}^{-1} \\
\text { calculated }\end{array}$ \\
\hline $\begin{array}{l}1 \\
1 \\
5 \\
1 \\
5\end{array}$ & $\begin{array}{l}12,124 \\
11,996.8 \\
11,819.3 \\
11,611.7 \\
11,457.4\end{array}$ & $\begin{array}{l}8,245.9 \\
8,333.28 \\
8,458.43 \\
8,609.65 \\
8,725.60\end{array}$ & $\begin{array}{l}1 s_{2}-2 p_{5} \\
3 d_{3}-4 \mathrm{Y} ? \\
2 p_{10}-2 s_{5} ? \\
2 s_{4}-5 \mathrm{Y} \\
2 p_{10}-2 s_{4}\end{array}$ & $\begin{array}{l}8,246.153 \\
8,333.64 \\
8,458.43 \\
8,609.56 \\
8,725.60\end{array}$ \\
\hline $\begin{array}{r}3 \\
6 \\
3 \\
20 \\
5\end{array}$ & $\begin{array}{l}11,259.0 \\
11,257.7 \\
11,187.0 \\
10,874.84 \\
10,699.2\end{array}$ & $\begin{array}{l}\text { 8, 879. } 35 \\
8,880.38 \\
8,936.50 \\
9,193.02 \\
9,343.93\end{array}$ & $\begin{array}{l}3 d_{5}-4 \mathrm{X} \\
3 d_{5}-4 \mathrm{Z} \\
3 d_{5}-4 \mathrm{Y} \\
3 d_{6}-4 \mathrm{X} \\
3 d_{4}^{\prime}-4 p_{9}\end{array}$ & $\begin{array}{l}8,879.48 \\
8,880.28 \\
8,936.30 \\
9,193.02 \\
9,343.62\end{array}$ \\
\hline $\begin{array}{r}3 \\
5 \\
30 \\
1 \\
1\end{array}$ & $\begin{array}{l}10,626.5 \\
10,608.37 \\
10,592.97 \\
10,575.48 \\
10,486.28\end{array}$ & $\begin{array}{l}9,407.86 \\
9,423.94 \\
9,437.64 \\
9,453.25 \\
9,533.66\end{array}$ & $\begin{array}{l}3 d_{1}^{\prime}-5 \mathrm{U} \\
3 d_{1}^{\prime}-5 \mathrm{Y} \\
3 d_{1}^{\prime}-5 \mathrm{~W} \\
3 d_{3}-4 p_{8} \\
3 d_{3}-4 p_{7}\end{array}$ & $\begin{array}{l}9,407.80 \\
9,424.32 \\
9,437.71 \\
9,453.07 \\
9,533.60\end{array}$ \\
\hline $\begin{array}{r}5 \\
10 \\
100 \\
2 \\
80\end{array}$ & $\begin{array}{l}10,458.56 \\
10,374.44 \\
10,360.37 \\
10,322.88 \\
10,296.93\end{array}$ & $\begin{array}{l}9,558.93 \\
9,636.44 \\
9,649.52 \\
9,684.56 \\
9,708.97\end{array}$ & $\begin{array}{l}3 d_{3}-4 p_{6} \\
3 d_{1}^{\prime \prime}-5 \mathrm{Y} \\
3 d_{1}^{\prime \prime}-5 \mathrm{~W} \\
2 p_{3}-5 d_{6} \\
2 p_{5}-4 d_{5}\end{array}$ & $\begin{array}{l}9,558.97 \\
9,636.15 \\
9,649.54 \\
9,684.47 \\
9,708.904\end{array}$ \\
\hline $\begin{array}{r}2 \\
10 \\
30 \\
10 \\
10\end{array}$ & $\begin{array}{l}10,273.6 \\
10,147.68 \\
10,120.96 \\
10,077.66 \\
10,065.96\end{array}$ & $\begin{array}{l}\text { 9, 831. } 02 \\
9,851.77 \\
9,877.78 \\
9,920.22 \\
9,931.75\end{array}$ & $\begin{array}{l}2 p_{2}-5 d_{5} \\
2 p_{2}-5 d_{3} \\
2 p_{3}-5 d_{3} \\
3 d_{5}-4 p_{10} \\
2 p_{2}-5 d_{4}\end{array}$ & $\begin{array}{l}9,831.00 \\
9,851.75 \\
9,877.77 \\
9,920.29 \\
9,931.739\end{array}$ \\
\hline $\begin{array}{l}2 \\
3 \\
1 \\
3 \\
4\end{array}$ & $\begin{array}{c}10,054.86 \\
10,038.65 \\
9,989.3 \\
9,917.60 \\
9,916.37\end{array}$ & $\begin{array}{c}9,942.72 \\
9,958.77 \\
10,008.0 \\
10,080.32 \\
10,081.57\end{array}$ & $\begin{array}{l}2 s_{4}-6 Z \\
2 s_{4}-6 Y ? \\
2 p_{4}-5 d_{6} \\
2 p_{4}-5 d_{5} \\
3 d_{1}^{\prime}-5 p_{6}\end{array}$ & $\begin{array}{r}9,942.53 \\
9,958.18 \\
10,007.70 \\
10,080.25 \\
10,081.47\end{array}$ \\
\hline
\end{tabular}

18 W. Gremmer, Zeitschr. f. Phys., vol. 54, p. 199, 1929.

19 W. F. Meggers, T. L. de Bruin, and C.J. Humphreys, B. S. Jour. of Research, vol. 3 (RP89), p. 129, 1929.

${ }_{20}$ W. F. Meggers, T. L. de Bruin, and C. J. Humphreys, B. S. Jour. of Research, vol. 7 (RP364), p. 643, 1931.

21 W. Gremmer, Zeitschr. f. Phys., vol. 73, p. 620, 1932.

22 E. Rasmussen, Zeitschr. f. Phys., vol. 73, p. 779, 1932. 
TABLE 5.-Infra-red spectrum of krypton-Continued

\begin{tabular}{|c|c|c|c|c|}
\hline Intensity & $\lambda_{\text {Bir I. A. }}$ I. & $\begin{array}{l}\nu_{\mathrm{vac}} \mathrm{cm}^{-1} \\
\text { observed }\end{array}$ & $\begin{array}{l}\text { Term combi- } \\
\text { nation }\end{array}$ & $\begin{array}{l}\nu_{\mathrm{vao}} \mathrm{cm}^{-1} \\
\text { calculated }\end{array}$ \\
\hline 2 & $9,897.08$ & $10,101.22$ & $2 p_{2}-5 d_{1}^{\prime}$ & $10,101.18$ \\
\hline 4 & $9,862.95$ & $10,136.18$ & $3 d_{5}-4 p_{7}$ & $10,136.26$ \\
\hline 500 & $9,856.24$ & $10,143.08$ & $2 p_{6}-3 s_{1}^{\prime \prime \prime \prime}$ & $10,143.06$ \\
\hline 5 & $9,838.33$ & $10,161.54$ & $3 d_{5}-4 p_{6}$ & $10,161.63$ \\
\hline $2 \mathrm{~h}$ & $9,810.27$ & $10,190.61$ & $3 d_{2}-6 \mathrm{X}$ & $10,190.60$ \\
\hline 3 & $9,794.89$ & $10,206.60$ & $3 d_{2}-6 \mathrm{Y}$ & $10,206.61$ \\
\hline 2 & $9,768.69$ & $10,233.98$ & $3 d_{\sigma}-4 p_{10}$ & $10,233.83$ \\
\hline 2,000 & $9,751.74$ & $10,251.77$ & $1 s_{4}-2 p_{10}$ & $10,251.750$ \\
\hline 50 & $9,743.11$ & $10,260.85$ & $3 d_{4}-5 U$ & $10,260.80$ \\
\hline $2 \mathrm{~h}$ & $9,727.51$ & $10,277.31$ & $3 d_{4}-5 \mathrm{Y}$ & $10,277.32$ \\
\hline 1 & $9,722.78$ & $10,282.30$ & $3 d_{1}^{\prime \prime}-5 p_{7}$ & $10,281.85$ \\
\hline 15 & $9,714.85$ & $10,290.70$ & $3 d_{4}-5 \mathrm{~W}$ & $10,290.71$ \\
\hline 50 & $9,704.22$ & $10,301.97$ & $2 p_{T}-3 s_{1}{ }^{\prime \prime \prime \prime}$ & $10,302.01$ \\
\hline 10 & $9,687.83$ & $1.0,319.40$ & $2 p_{\sigma}-3 s_{1}^{\prime \prime}$ & $10,319.35$ \\
\hline 2 & $9,682.26$ & $10,325.34$ & $3 d_{5}-4 p_{5}$ & $10,325.32$ \\
\hline 1 & $9,669.03$ & $10,339.47$ & $2 p_{3}-5 d_{2}$ & $10,339.64$ \\
\hline 3 & $9,615.63$ & $10,396.88$ & $2 p_{4}-5 d_{1}^{\prime \prime}$ & $10,396.89$ \\
\hline $\begin{array}{r}1 \\
30\end{array}$ & $\begin{array}{l}9,607.2 \\
9,540.89\end{array}$ & $\begin{array}{l}10,406.0 \\
10,478.33\end{array}$ & $2 p_{\tau}-3 s_{1}^{\prime \prime}$ & $10.478,30$ \\
\hline 1 & $9,532.3$ & $10,487.8$ & $2 p_{1}-6 d_{5}$ & $10,487.87$ \\
\hline 20 & $9,450.88$ & $10,578.13$ & $2 p_{6}-3 s_{1}^{\prime \prime \prime}$ & 1p, 578. 09 \\
\hline 100 & $9,362.03$ & $10,678.52$ & $2 p_{6}-4 \bar{d}_{5}$ & $10,678.428$ \\
\hline 100 & $9,352.23$ & $10,689.61$ & $3 d_{4}^{\prime}-5 \mathrm{U}$ & $10,689.72$ \\
\hline 1 & $9,337.9$ & $10,706.1$ & $3 d_{4}^{\prime}-5 \mathrm{Y}$ & $10,706.24$ \\
\hline 10 & $9,326.03$ & $10,719.74$ & $3 d_{4}^{\prime}-5 \mathrm{~W}$ & $10,719.63$ \\
\hline 1 & $9,299.40$ & $10,750.43$ & $3 d_{3}-3 p_{4}$ & $10,750.58$ \\
\hline 1 & $9,287.87$ & $10,763.78$ & $3 d_{1}^{\prime}-6 U$ & $10,763.85$ \\
\hline 2 & $9,279.9$ & $10,773.0$ & $3 d_{1}^{\prime}-6 \mathrm{Y}$ & $10,772.94$ \\
\hline 8 & $9,273.02$ & $10,781.02$ & $3 d_{1}^{\prime}-6 \mathrm{~W}$ & $10,781.04$ \\
\hline 10 & $9,270.96$ & $10,783.41$ & $3 d_{3}-5 Z$ & $10,783.41$ \\
\hline 1 & $9,262.69$ & $10,793.04$ & $3 d_{3}-5 \mathrm{X}$ & $10,793.04$ \\
\hline 30 & $9,243.54$ & $10,815.40$ & $2 \pi$ EV & \\
\hline 1 & $9,243.00$ & $\begin{array}{l}10,816.03 \\
10,826,39\end{array}$ & $\begin{array}{l}3 d_{3}-5 Y \\
3 d_{3}-3 p_{3}\end{array}$ & $\begin{array}{l}10,815.97 \\
10.826 .50\end{array}$ \\
\hline 1 & $9,224.83$ & $10,837.34$ & $2 p_{T}-4 d_{5}$ & $10,837.372$ \\
\hline 2 & $9,188.69$ & $10,879.96$ & $3 d_{3}-3 p_{2}$ & $10,880.09$ \\
\hline 20 & $9,122.49$ & $10,958.91$ & $2 p_{8}-3 s_{1}^{\prime \prime \prime \prime}$ & $10,959.02$ \\
\hline 20 & $9,111.69$ & $10,971.90$ & $2 p_{9}-3 s_{1}^{\prime \prime \prime \prime}$ & $10,972.00$ \\
\hline 1 & $9,100.58$ & $10,985.30$ & $3 d_{1}^{\prime \prime}-6 \mathrm{Y}$ & $10,984.77$ \\
\hline $4 \mathrm{~h}$ & $9,094.33$ & $10,992.85$ & $3 d_{1}^{\prime \prime}-6 \mathrm{~W}$ & $10,992.87$ \\
\hline 3 & $9,044.47$ & $11,053.45$ & $2 p_{5}-3 s_{1}{ }^{\prime}$ & $11,053.49$ \\
\hline 30 & $8,999.19$ & $11,109.06$ & $2 p_{T}-4 d_{6}$ & $11,109.076$ \\
\hline $1 ?$ & $8,978.5$ & $11,134.6$ & $3 d_{3}-3 p_{1} ?$ & $11,133.89$ \\
\hline 50 & $8,977.99$ & $11,135.20$ & $2 p_{8}-3 s_{1}{ }^{\prime \prime}$ & $11,135.31$ \\
\hline 10 & $8,967.53$ & $11,148.29$ & $2 p_{0}-3 s_{1}^{\prime \prime}$ & $11,148.29$ \\
\hline 2,000 & 8, 928. 6934 & $11,196.7764$ & $1 s_{5}-2 p_{10}$ & $11,196.776$ \\
\hline 2 & $8,925.3$ & $11,201.0$ & & \\
\hline $\begin{array}{l}4 \\
3\end{array}$ & $\begin{array}{l}8,870.32 \\
8,842.46\end{array}$ & $\begin{array}{l}11,270.46 \\
11,305.97\end{array}$ & $3 d_{1}^{\prime}-5 p_{9}$ & $11,305.63$ \\
\hline 20 & $8,805.78$ & $11,353.06$ & $3 d_{5}-3 p_{4}$ & $11,353.24$ \\
\hline
\end{tabular}


TABLE 5.-Infra-red spectrum of krypton-Continued

\begin{tabular}{|c|c|c|c|c|}
\hline Intensity & $\lambda_{\text {air }}$ I. A. & $\begin{array}{l}\nu_{\mathrm{VBo}} \mathrm{cm}^{-1} \\
\text { observed }\end{array}$ & $\begin{array}{l}\text { Term combi- } \\
\text { nation }\end{array}$ & $\begin{array}{l}\nu_{\mathrm{vao}} \mathrm{cm}^{-1} \\
\text { calculated }\end{array}$ \\
\hline $\begin{array}{r}30 \\
6,000 \\
50 \\
4 \\
150\end{array}$ & $\begin{array}{l}\text { 8, 780. } 25 \\
8,776.7498 \\
8,774.05 \\
8,773.00 \\
8,764.09\end{array}$ & $\begin{array}{l}11,386.07 \\
11,390.6150 \\
11,394.12 \\
11,395.48 \\
11,407.08\end{array}$ & $\begin{array}{l}3 d_{5}-5 Z \\
1 s_{4}-2 p_{8} \\
2 p_{8}-3 s_{1}^{\prime \prime \prime} \\
3 d_{5}-5 \mathrm{X} \\
2 p_{9}-3 s_{1}{ }^{\prime \prime \prime}\end{array}$ & $\begin{array}{l}11,386.07 \\
11,390.615 \\
11,394.05 \\
11,395.70 \\
11,407.03\end{array}$ \\
\hline $\begin{array}{r}30 \\
2 \\
3 \\
1 \\
8\end{array}$ & $\begin{array}{l}8,755.20 \\
8,747.29 \\
8,746.43 \\
8,742.49 \\
8,726.54\end{array}$ & $\begin{array}{l}11,418.65 \\
11,428.97 \\
11,430.10 \\
11,435.25 \\
11,456.15\end{array}$ & $\begin{array}{l}3 d_{5}-5 Y \\
3 d_{5}-3 p_{3} \\
2 p_{2}-6 d_{3} \\
2 p_{1}-7 d_{5} \\
2 p_{3}-6 d_{3}\end{array}$ & $\begin{array}{l}11,418.63 \\
11,429.16 \\
11,430.17 \\
11,435.27 \\
11,456.19\end{array}$ \\
\hline $\begin{array}{r}1 \\
2 \\
40 \\
2 \\
8\end{array}$ & $\begin{array}{l}8,722.17 \\
8,713.62 \\
8,697.50 \\
8,673.48 \\
8,651.49\end{array}$ & $\begin{array}{l}11,461.76 \\
11,473.14 \\
11,494.40 \\
11,526.23 \\
11,555.66\end{array}$ & $\begin{array}{l}3 d_{3}-5 p_{7} \\
3 d_{3}-5 p_{6} \\
2 p_{8}-4 d_{5} \\
2 p_{2}-6 d_{4} \\
2 p_{5}-4 d_{2}\end{array}$ & $\begin{array}{l}11,461.85 \\
11,473.16 \\
11,494.389 \\
11,526.30 \\
11,555.57\end{array}$ \\
\hline $\begin{array}{l}1 \\
1 \\
1 \mathrm{~h} \\
4 \mathrm{~h} \\
40\end{array}$ & $\begin{array}{l}8,632.81 \\
8,631.5 \\
8,628.70 \\
8,624.82 \\
8,605.85\end{array}$ & $\begin{array}{l}11,580.54 \\
11,582.3 \\
11,586.05 \\
11,591.26 \\
11,616.81\end{array}$ & $\begin{array}{l}3 d_{1}{ }^{\prime}-7 \mathrm{U} \\
2 p_{2}-6 d_{1}^{\prime \prime} \\
3 d_{1}^{\prime}-7 \mathrm{Y} \\
3 d_{1}^{\prime}-7 \mathrm{~W} \\
3 d_{4}-6 \mathrm{U}\end{array}$ & $\begin{array}{l}11,580.58 \\
11,582.39 \\
11,586.06 \\
11,591.35 \\
11,616.85\end{array}$ \\
\hline $\begin{array}{r}1 \\
10 \\
20 \\
50 \\
40\end{array}$ & $\begin{array}{l}8,599.4 \\
8,593.1 \\
8,569.02 \\
8,560.89 \\
8,537.93\end{array}$ & $\begin{array}{l}11,625.5 \\
11,634.05 \\
11,666.74 \\
11,677.82 \\
11,709.23\end{array}$ & $\begin{array}{l}3 d_{4}-6 \mathrm{Y} \\
3 d_{4}-6 \mathrm{~W} \\
3 d_{6}-3 p_{4} \\
2 p_{5}-3 s_{4} \\
3 d_{6}-5 \mathrm{X}\end{array}$ & $\begin{array}{l}11,625.94 \\
11,634.04 \\
11,666.78 \\
11,677.83 \\
11,709.24\end{array}$ \\
\hline $\begin{array}{r}3,000 \\
30 \\
2 \\
2 \mathrm{~h} \\
100\end{array}$ & $\begin{array}{l}8,508.8736 \\
8,498.21 \\
8,477.20 \\
8,469.96 \\
8,412.45\end{array}$ & $\begin{array}{l}11,749.2128 \\
11,763.96 \\
11,793.11 \\
11,803.19 \\
11,883.89\end{array}$ & $\begin{array}{l}1 s_{2}-2 p_{4} \\
2 p_{6}-2 s_{2} \\
2 p_{6}-4 d_{4} \\
3 d_{1}{ }^{\prime \prime}-7 \mathrm{~W} \\
2 p_{6}-4 d_{3}\end{array}$ & $\begin{array}{l}11,749213 \\
11,763.975 \\
11,793.139 \\
11,803.18 \\
11,883.903\end{array}$ \\
\hline $\begin{array}{r}15 \\
5 \\
1 \\
2 \\
10\end{array}$ & $\begin{array}{l}8,384.90 \\
8,375.93 \\
8,332.73 \\
8,321.09 \\
8,303.20\end{array}$ & $\begin{array}{l}11,922.93 \\
11,935.70 \\
11,997.58 \\
12,014.36 \\
12,040.24\end{array}$ & $\begin{array}{l}2 p_{\tau}-2 s_{2} \\
3 d_{5}-5 p_{10} \\
2 p_{6}-4 d_{1}^{\prime \prime}\end{array}$ & $\begin{array}{l}11,922.919 \\
11,997.67 \\
12,040.15\end{array}$ \\
\hline $\begin{array}{r}20 \\
5,000 \\
4 \mathrm{~h} \\
1,500 \\
100\end{array}$ & $\begin{array}{l}\text { 8, 301. 39 } \\
8,298.1091 \\
8,287.56 \\
8,281.05 \\
8,272.36\end{array}$ & $\begin{array}{l}12,042.87 \\
12,047.6312 \\
12,062.96 \\
12,072.45 \\
12,085.13\end{array}$ & $\begin{array}{l}2 p_{7}-4 d_{3} \\
1 s_{4}-2 p_{7} \\
3 d_{4}^{\prime}-6 \mathrm{~W} \\
1 s_{2}-2 p_{3} \\
2 p_{6}-4 d_{1}^{\prime}\end{array}$ & $\begin{array}{l}12,042.847 \\
12,047.632 \\
12,062.96 \\
12,072.446 \\
12,085.16\end{array}$ \\
\hline $\begin{array}{c}3,000 \\
10 \mathrm{~h} \\
6 \\
80 \\
1\end{array}$ & $\begin{array}{l}8,263.2412 \\
8,228.89 \\
8,222.69 \\
8,218.40 \\
8,210.1\end{array}$ & $\begin{array}{l}12,098.4676 \\
12,148.97 \\
12,158.13 \\
12,164.48 \\
12,176.8\end{array}$ & $\begin{array}{l}1 s_{2}-2 p_{2} \\
3 d_{3}-6 \mathrm{X} \\
2 p_{2}-4 s_{1}^{\prime} \\
3 d_{3}-6 Y^{\prime \prime} \\
2 p_{2}-4 s_{1}^{\prime \prime}\end{array}$ & $\begin{array}{l}12,098.465 \\
12,148.58 \\
12,158.08 \\
12,164.59 \\
12,176.91\end{array}$ \\
\hline $\begin{array}{r}40 \\
20 \\
50 \\
2 \\
3,000\end{array}$ & $\begin{array}{l}8,206.62 \\
8,205.22 \\
8,195.07 \\
8,192.4 \\
8,190.0570\end{array}$ & $\begin{array}{l}12,181 \cdot 94 \\
12,184.02 \\
12,199.11 \\
12,203.1 \\
12,206.5759\end{array}$ & $\begin{array}{l}2 p_{\tau}-3 s_{1}{ }^{\prime} \\
2 p_{3}-4 s_{1}{ }^{\prime} \\
2 p_{\tau}-4 d_{1}{ }^{\prime \prime} \\
2 p_{3}-4 s_{1}^{\prime \prime} \\
1 s_{1}-2 p_{6}\end{array}$ & $\begin{array}{l}12,181.96 \\
12,184.10 \\
12,199.10 \\
12,202.93 \\
12,206.576\end{array}$ \\
\hline
\end{tabular}


TABLE 5.-Infra-red spectrum of krypton-Continued

\begin{tabular}{|c|c|c|c|c|}
\hline Intensity & $\lambda_{\text {air I. A. }}$. & $\begin{array}{l}\nu_{\mathrm{vac}} \mathrm{cm}^{-1} \\
\text { observed }\end{array}$ & $\begin{array}{l}\text { Term combi- } \\
\text { nation }\end{array}$ & $\begin{array}{l}\nu_{\mathrm{vac}} \mathrm{cm}^{-1} \\
\text { calculated }\end{array}$ \\
\hline $\begin{array}{r}15 \\
60 \\
6,000 \\
4,000 \\
500\end{array}$ & $\begin{array}{l}8,144.96 \\
8,132.98 \\
8,112.9023 \\
8,104.3660 \\
8,104.02\end{array}$ & $\begin{array}{l}12,274.16 \\
12,292.24 \\
12,322.6615 \\
12,335.6408 \\
12,336.17\end{array}$ & $\begin{array}{l}2 p_{10}-3 s_{1}{ }^{\prime \prime} \\
2 p_{2}-4 s_{1}{ }^{\prime \prime \prime} \\
1 s_{5}-2 p_{9} \\
1 s_{5}-2 p_{8} \\
2 p_{9}-4 d_{4}{ }^{\prime}\end{array}$ & $\begin{array}{ll}12,274 . & 18 \\
12,292 . & 40 \\
12,322 . & 662 \\
12,335 . & 641 \\
12,336 . & 17\end{array}$ \\
\hline $\begin{array}{c}1,500 \\
8 h \\
1 \\
2 h \\
5\end{array}$ & $\begin{array}{l}8,059.5053 \\
8,040.50 \\
8,039.8 \\
8,033.52 \\
7,993.12\end{array}$ & $\begin{array}{l}12,404.3030 \\
12,433.62 \\
12,434.7 \\
12,444.43 \\
12,507.32\end{array}$ & $\begin{array}{l}1 s_{3}-2 p_{4} \\
3 d_{4}-7 \mathrm{U} \\
\\
3 d_{4}-7 \mathrm{~W} \\
2 p_{4}-4 s_{1}^{\prime}\end{array}$ & $\begin{array}{l}12,404.302 \\
12,433.58 \\
12,444.35 \\
12,507.33\end{array}$ \\
\hline $\begin{array}{r}2 \\
100 \\
30 \\
20 \\
1\end{array}$ & $\begin{array}{l}7,990.78 \\
7,982.42 \\
7,981.82 \\
7,981.19 \\
7,962.62\end{array}$ & $\begin{array}{l}12,510.99 \\
12,524.08 \\
12,525.05 \\
15,526.02 \\
12,555.23\end{array}$ & $\begin{array}{l}2 p_{6}-3 s_{5} \\
2 p_{6}-4 d_{2} \\
2 p_{4}-4 s_{1}{ }^{\prime \prime} \\
3 d_{3}-6 p_{6}\end{array}$ & $\begin{array}{l}12,524.142 \\
12,525.09 \\
12,526.16 \\
12,555.12\end{array}$ \\
\hline $\begin{array}{r}2 \\
20 \\
2 \\
180 \\
40\end{array}$ & $\begin{array}{l}7,957.67 \\
7,946.99 \\
7,938.34 \\
7,928.602 \\
7,920.47\end{array}$ & $\begin{array}{l}12,563.04 \\
12,579.92 \\
12,593.63 \\
12,609.100 \\
12,622.05\end{array}$ & $\begin{array}{l}2 p_{2}-7 d_{1}{ }^{\prime} \\
2 p_{\mathrm{s}}-2 s_{2} \\
2 p_{3}-7 d_{3} \\
2 p_{8}-4 d_{4} \\
2 p_{9}-4 d_{4}\end{array}$ & $\begin{array}{l}12,562.92 \\
12,579.936 \\
12,593.62 \\
12,609.100 \\
12,622.079\end{array}$ \\
\hline $\begin{array}{r}200 \\
30 \\
10 \\
30 \\
2\end{array}$ & $\begin{array}{l}7,913.443 \\
7,904.62 \\
7,882.36 \\
7,881.76 \\
7,871.93\end{array}$ & $\begin{array}{l}12,633.254 \\
12,647.35 \\
12,683.07 \\
12,684.03 \\
12,699.88\end{array}$ & $\begin{array}{l}2 p_{10}-4 d_{5} \\
2 p_{6}-3 s_{4} \\
2 p_{7}-3 s_{5} \\
2 p_{\tau}-4 d_{2} \\
2 p_{8}-4 d_{3}\end{array}$ & $\begin{array}{l}12,633.254 \\
12,647.35 \\
12,683.086 \\
12,684.04 \\
12,699.864\end{array}$ \\
\hline $\begin{array}{r}20 \\
800 \\
4 \\
8 h \\
2 h\end{array}$ & $\begin{array}{l}7,863.91 \\
7,854.823 \\
7,840.40 \\
7,840.01 \\
7,830.21\end{array}$ & $\begin{array}{l}12,712.81 \\
12,727.535 \\
12,750.96 \\
12,751.58 \\
12,767.54\end{array}$ & $\begin{array}{l}2 p_{9}-4 d_{3} \\
1 s_{3}-2 p_{3} \\
3 d_{5}-6 \mathrm{X} \\
3 d_{5}-6 \mathrm{Z} \\
3 d_{5}-6 \mathrm{Y}\end{array}$ & $\begin{array}{l}12,712.843 \\
12,727.535 \\
12,751.24 \\
12,751.60 \\
12,767.25\end{array}$ \\
\hline $\begin{array}{c}50 \\
2 \\
40 \\
5 h \\
5\end{array}$ & $\begin{array}{l}7,806.52 \\
7,786.66 \\
7,776.28 \\
7,772.40 \\
7,768.43\end{array}$ & $\begin{array}{l}12,806.29 \\
12,838.95 \\
12,856.09 \\
12,862.50 \\
12,869.08\end{array}$ & $\begin{array}{l}2 p_{T}-3 s_{4} \\
2 p_{s}-3 s_{1}{ }^{\prime} \\
2 p_{8}-4 d_{1}{ }^{\prime \prime} \\
3 d^{\prime}-7 U^{\prime} \\
2 p_{9}-4 d_{1}^{\prime \prime}\end{array}$ & $\begin{array}{l}12,806.30 \\
12,838.97 \\
12,856.11 \\
12,862.50 \\
12,869.09\end{array}$ \\
\hline $\begin{array}{c}1 \\
3 \\
150 \\
40 \\
1 h\end{array}$ & $\begin{array}{l}7,765.89 \\
7,749.16 \\
7,746.831 \\
7,741.39 \\
7,712.0\end{array}$ & $\begin{array}{l}12,873.29 \\
12,901.08 \\
12,904.958 \\
12,914.03 \\
12,963.2\end{array}$ & $\begin{array}{l}3 d^{\prime}{ }_{4}-7 \mathrm{~W} \\
2 p_{8}-4 d_{1}{ }^{\prime} \\
2 p_{10}-4 d_{6} \\
2 p_{9}-4 d_{1}{ }^{\prime} \\
3 d_{4}-8 U^{\prime}\end{array}$ & $\begin{array}{l}12,873.27 \\
12,901.12 \\
12,904.958 \\
12,914.10 \\
12,963.7\end{array}$ \\
\hline $\begin{array}{r}1 h \\
2 h \\
1,200 \\
1,000 \\
4\end{array}$ & $\begin{array}{l}7,709.0 \\
7,703.41 \\
7,694.5401 \\
7,685.2472 \\
7,652.16\end{array}$ & $\begin{array}{l}12,968.3 \\
12,977.70 \\
12,992.6577 \\
13,008.3682 \\
13,064.62\end{array}$ & $\begin{array}{l}3 d_{3}-7 Z \\
3 d_{3}-7 \mathrm{Y} \\
1 s_{5}-2 p_{7} \\
1 s_{2}-2 p_{1} \\
3 d_{6}-6 \mathrm{X}\end{array}$ & $\begin{array}{l}12,968.1 \\
12,977.71 \\
12,992.658 \\
13,008.368 \\
13,064.78\end{array}$ \\
\hline 4,000 & $7,601.5465$ & $13,151.6027$ & $1 s_{5}-2 p_{6}$ & $13,151.602$ \\
\hline
\end{tabular}




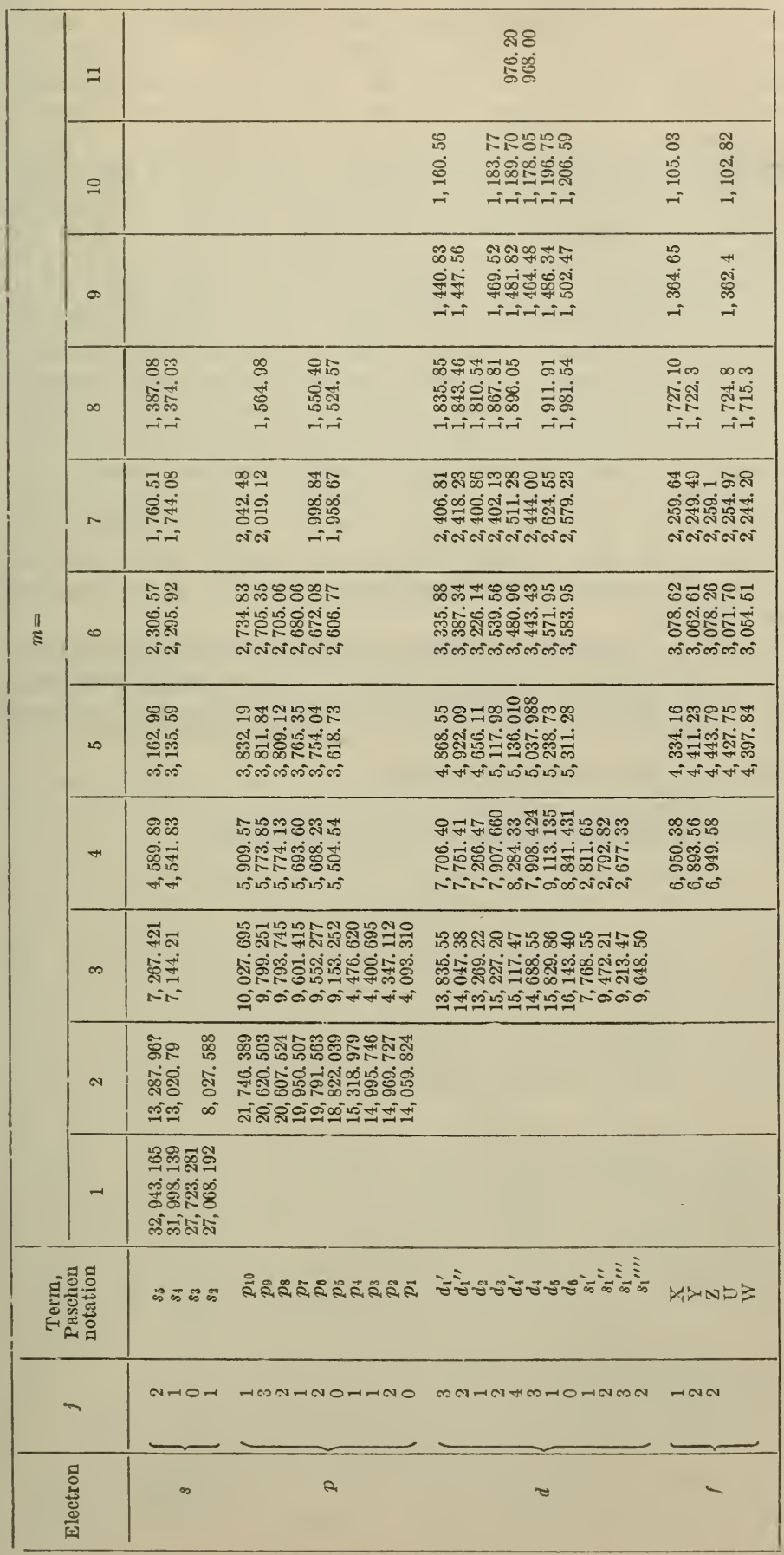


The present investigation of the infra-red spectrum of krypton with mesocyanine and xenocyanine plates yields 200 lines, more than half of which have never been observed before. Analysis of these data brings the discovery of spectral terms nearer completion and firmly establishes the nature of some terms previously known but misinterpreted on account of unobserved combinations. The corrections suggested by Gremmer are justified, and most of the extensions and revisions of terms by Rasmussen are confirmed. The results for the observed and classified lines of krypton are presented in Table 5 in the same manner as they were shown for neon and argon in Tables 1 and 3 , respectively. Wave lengths given to more than 2 decimal places are quoted from the interferometer measurements by Humphreys. ${ }^{23}$ Such values fix the relative values of combining terms very accurately and thus permit the calculation of other wave lengths with somewhat higher precision than can be claimed for ordinary grating measurements. A complete list of the $\mathrm{Kr}$ I terms now available is given in Table 6, which is comparable with Tables 2 and 4 for Ne I and A I spectra, respectively. The $2 s_{4}$ term at $13,020.79$ is checked by double-electron $(s-f)$ transitions, but no such combinations were observed for the $2 s_{5}$ term, which was placed at $13,287.96$ on the assumption that the otherwise unclassified line at 8,458.43 A represented the transition $2 p_{10}-2 s_{5}$.

Radiometric investigation of this spectrum is recommended to those equipped to undertake it because many of the expected combinations of known terms still lie beyond the range of present photographic materials.

Washington, November 26, 1932.

23 C. J. Humphreys, B. S. Jour. of Research, vol. 5 (R P 245), p. $1041 ; 1930$. 